ARTICLE

https://doi.org/10.1038/s41467-019-13174-6

\title{
JASPer controls interphase histone H3S10 phosphorylation by chromosomal kinase JIL-1 in Drosophila
}

Christian Albig (iD) ${ }^{1,2}$, Chao Wang ${ }^{3}$, Geoffrey P. Dann ${ }^{4,6}$, Felix Wojcik ${ }^{4}$, Tamás Schauer ${ }^{5}$, Silke Krause ${ }^{1}$, Sylvain Maenner ${ }^{1,7}$, Weili Cai ${ }^{3}$, Yeran Li ${ }^{3}$, Jack Girton ${ }^{3}$, Tom W. Muir (1) 4, Jørgen Johansen (1) ${ }^{3}$, Kristen M. Johansen (i) ${ }^{3}$, Peter B. Becker (1) ${ }^{1 \star} \&$ Catherine Regnard (10) ${ }^{1 \star}$

In flies, the chromosomal kinase JIL-1 is responsible for most interphase histone H3S10 phosphorylation and has been proposed to protect active chromatin from acquiring heterochromatic marks, such as dimethylated histone H3K9 (H3K9me2) and HP1. Here, we show that JIL-1's targeting to chromatin depends on a PWWP domain-containing protein JASPer (JIL-1 Anchoring and Stabilizing Protein). JASPer-JIL-1 (JJ)-complex is the major form of kinase in vivo and is targeted to active genes and telomeric transposons via binding of the PWWP domain of JASPer to H3K36me3 nucleosomes, to modulate transcriptional output. $\mathrm{JIL-1}$ and JJ-complex depletion in cycling cells lead to small changes in H3K9me2 distribution at active genes and telomeric transposons. Finally, we identify interactors of the endogenous JJ-complex and propose that JIL-1 not only prevents heterochromatin formation but also coordinates chromatin-based regulation in the transcribed part of the genome.

\footnotetext{
${ }^{1}$ Molecular Biology Division, Biomedical Center, Faculty of Medicine and Center for Integrated Protein Science Munich (CIPSM), LMU Munich, 82152 Martinsried, Germany. ${ }^{2}$ Graduate School for Quantitative Biosciences (QBM), LMU Munich, 81377 Munich, Germany. ${ }^{3}$ Roy J. Carver Department of Biochemistry, Biophysics, and Molecular Biology, lowa State University, Ames, IA 50011, USA. ${ }^{4}$ Department of Chemistry, Frick Laboratory, Princeton University, Princeton, NJ 08544, USA. ${ }^{5}$ Bioinformatics Unit, Biomedical Center, Faculty of Medicine, LMU Munich, 82152 Martinsried, Germany. ${ }^{6}$ Present address: Department of Biochemistry and Biophysics, Perelman School of Medicine, University of Pennsylvania, Philadelphia, PA 19104, USA. 7Present address: UMR7365 CNRS-UL, IMoPA, University of Lorraine, 54505 Vandoeuvre-lès-Nancy, France. *email: pbecker@bmc.med.Imu.de; cregnard@bmc.med.Imu.de
} 
n mammals, several nuclear kinases contribute to phosphorylation of histone $\mathrm{H} 3$ at serine $10(\mathrm{H} 3 \mathrm{~S} 10 \mathrm{ph})$ in interphase, whereas in Drosophila melanogaster, the essential kinase JIL-1 is responsible for most of $i^{1}$. The significance of interphase $\mathrm{H} 3 \mathrm{~S} 10 \mathrm{ph}$ is often underestimated because most H3S10 phosphorylation in asynchronous cell populations stems from mitotic chromatin, where it is deployed by Aurora B kinase ${ }^{2,3}$. Originally, interphase $\mathrm{H} 3 \mathrm{~S} 10 \mathrm{ph}$ has been associated, in combination with $\mathrm{H} 3 \mathrm{~K} 9 \mathrm{ac}$ and $\mathrm{H} 3 \mathrm{~K} 14 \mathrm{ac}$, with transcriptional activation of immediate early genes upon MAPK activation ${ }^{4,5}$. In Drosophila, interphase $\mathrm{H} 3 \mathrm{~S} 10 \mathrm{ph}$ is enriched at the body of active genes ${ }^{6}$. In mammal, in the extreme case of mouse embryonic stem cells (mESC), $\sim 30 \%$ of the genome is enriched for H3S10ph in interphase ${ }^{7}$.

The current model assigns JIL-1 to the protection of euchromatin from heterochromatization ${ }^{8}$. According to the phosphomethyl switch model for mitotic H3S10ph', placing H3S10ph prevents $\mathrm{H} 3 \mathrm{~K} 9$ methylation and subsequent binding of heterochromatin components. JIL-1 phosphorylates various $\mathrm{H} 3$ peptides with different methylation states, including $\mathrm{H} 3 \mathrm{~K} 9 \mathrm{me} 2 / 3$, with comparable efficiency ${ }^{6}$, whereas histone methyltransferases of the $\mathrm{Su}$ (var)3-9 family are inhibited by H3S10 $\mathrm{ph}^{10,11}$. Several observations suggest that JIL-1 is important for the balance between euchromatin and heterochromatin. The Su(var)3-1 alleles of JIL-1 gene, which lead to the expression of JIL-1 truncated in its C-terminal domain (CTD), result in reduced heterochromatin spreading at euchromatin-heterochromatin boundaries $^{12,13}$. Conversely, in the $J I L-1^{z 2 / z 2}$ null mutant, heterochromatin components spread into euchromatin. The spreading of $\mathrm{H} 3 \mathrm{~K} 9 \mathrm{me} 2$ and $\mathrm{HP} 1$ is highest on the euchromatic part of the X chromosome in both sexes ${ }^{14}$, the spreading of the 7 zinc-finger protein $\mathrm{Su}$ (var)3-7 affects euchromatin similarly on all chromosomes $^{15}$. In addition, JIL-1 phosphorylates $\mathrm{Su}(\mathrm{var}) 3-9^{16}$, the histone methyl transferase responsible for $\mathrm{H} 3 \mathrm{~K} 9 \mathrm{me} 2 / 3$, suggesting a possible function for JIL-1 at constitutive heterochromatin.

JIL-1 may also play a role at telomeres, which combine features of heterochromatin and euchromatin in Drosophila. JIL-1 localizes to arrays composed of the three non-LTR retrotransposons HeT-A, TART, and TAHRE (HTT) on polytene chromosomes in mutants with elongated telomeres ${ }^{17}$. Transcription of HTT arrays is essential for telomere maintenance in flies, and JIL-1 is a positive regulator of retrotransposon transcription ${ }^{18,19}$.

At the low resolution of polytene chromosomes, JIL-1 localizes to active chromatin and is enriched on the male dosagecompensated $\mathrm{X}$ chromosome ${ }^{20}$. When the binding of JIL-1 to chromatin was studied at higher resolution using chromatinimmunoprecipitation (ChIP), conflicting results were obtained. Our early ChIP-chip study suggested that JIL-1 is found on all transcribed gene bodies and is enriched on X-chromosomal genes in male S2 cells ${ }^{6}$. ChIP-seq experiments from female Kc cells ${ }^{21}$ and salivary glands ${ }^{8}$ suggested that JIL- 1 associates to the 5 ' end/ promoters of active genes and to enhancers.

In this work, we show that the JIL-1 protein level is tightly controlled by JASPer (JIL-1 Anchoring and Stabilizing Protein), a PWWP domain-containing protein. Both proteins form a stable JASPer-JIL-1 (JJ)-complex, the functional form of the kinase in vivo. The PWWP domain of JASPer tethers the JJ-complex to $\mathrm{H} 3 \mathrm{~K} 36 \mathrm{me} 3$ nucleosomes in vitro. Consistently, the JJ-complex is targeted to H3K36me3 chromatin at active gene bodies and at telomeric transposons in vivo. Depletion of the JJ-complex in flies induces heterochromatin spreading in salivary gland nuclei as described for the JIL-1 deficiency. Using D. melanogaster cell lines, we show that depletion of JIL-1 or the JJ-complex modulates the transcriptional output. In male S2 cells, depletion of
JIL-1 results in a modest enrichment of $\mathrm{H} 3 \mathrm{~K} 9 \mathrm{me} 2$ in the active chromatin, where the JJ-complex binds. Finally, we identify various known and novel interactors of the endogenous JJ-complex, notably chromatin remodeling complexes and subunits of the Set1/COMPASS complex.

\section{Results}

JIL-1 forms a complex with the protein JASPer. Since JIL-1 lacks a known chromatin binding domain, we hypothesized that JIL-1 is recruited to chromatin by an interaction partner. To identify such a protein, we used nuclear extracts of D. melanogaster embryos to perform preparative immunoprecipitations (IPs) using antibodies against JIL-1. A protein of $\sim 60 \mathrm{kDa}$ copurified with JIL-1 using two different JIL-1 antibodies (Supplementary Fig. 1a). Mass spectrometry analysis identified the protein as encoded by the gene CG7946 on chromosome 3R. We named this protein 'JIL-1 Anchoring and Stabilizing Protein' (JASPer). Consistently, reverse IP's using antibodies against JASPer showed that JIL-1 was efficiently co-immunoprecipitated from embryo extracts and with similar efficiency (Fig. 1a). Coexpressing recombinant FLAG-JIL-1 and untagged JASPer ${ }^{22}$ yielded a stable complex (Fig. 1b). Coomassie-blue staining suggested a roughly equal stoichiometry for the recombinant and the endogenous complex (Fig. 1b, Supplementary Fig. 1a) (corresponding to a mass ratio of 2.6:1 at calculated molecular weights of $137 \mathrm{kDa}$ for JIL-1 and $53 \mathrm{kDa}$ for JASPer).

JASPer is a well-conserved protein among Drosophila species. It has an N-terminal PWWP domain and a C-terminal LEDGF/ IBD domain (Fig. 1c, Supplementary Figs. 2a, b). This PWWPLEDGF domain architecture is found in 94 eukaryotic proteins, with mostly unknown functions, except for the PSIP1/LEDGF chromatin adapter protein, which has pleiotropic functions in HIV infection and cancer development ${ }^{23,24}$. JIL-1 is also well conserved among distant Drosophila species (Supplementary Fig. 3), particularly in the N-terminal AGC kinase domain ${ }^{25}$, the C-terminal MAPK-related domain and its CTD. The CTD is rich in proline $(11 \%)$ and arginine $(9 \%)$ residues and most probably intrinsically disordered. Sequence comparison revealed a prionlike domain (PrlD) ${ }^{26}$ and putative PEST sequences ${ }^{27}$, which most probably relate to lower stability of the protein because of their intrinsic disorder ${ }^{28}$.

Using a LacO-LacI targeting system in flies, we found that LacI-JIL-1 full-length and LacI-JIL-1-CTD recruited endogenous JASPer to the LacO arrays, but JIL-1- $\Delta$ CTD did not (Supplementary Fig. 1b). We further mapped the interaction by coexpression and co-purification of various derivatives. Truncations in the CTD of JIL-1 were designed according to sequence conservation in Drosophilae JIL-1 homologs (Fig. 1c and Supplementary Fig. 3). Expression of the C-terminal deletion mutants of FLAG-JIL-1 (mutants a-g) with untagged, full-length JASPer showed that the minimal JASPer binding domain (JBD) encompasses 44 amino acids (982-1025) between the truncations $\mathrm{c}$ and $\mathrm{d}$ of the CTD (Fig. 1d). The JBD is rich in proline (22\%), glutamic acid (16\%), and aromatic residues tyrosine/phenylalanine $(16 \%)$. Furthermore, it contains a stretch of 7 conserved amino acids, DFxGFDE, matching the consensus motif (FxGF) found in proteins interacting with the LEDGF/IBD domain of PSIP1 ${ }^{29}$. Indeed, using various JASPer derivatives (Fig. 1c) coexpressed with full-length FLAG-JIL-1, we found that deletion of the 120 amino acids long LEDGF domain in the C-terminal half of JASPer ( $\triangle \mathrm{LEDGF}$ ) was sufficient to abrogate binding to JIL-1 (Fig. 1e). This domain contains a high proportion of charged residues (glutamic acid/aspartic acid: $18 \%$ and arginine/lysine residues: $17 \%)$. 
a

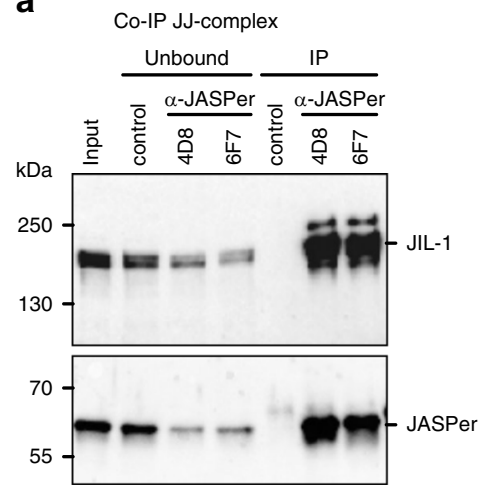

b

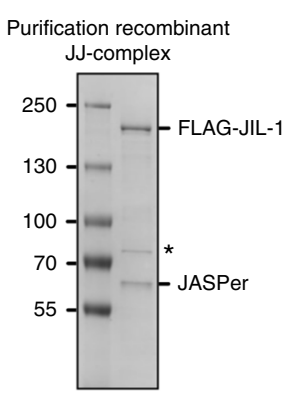

C

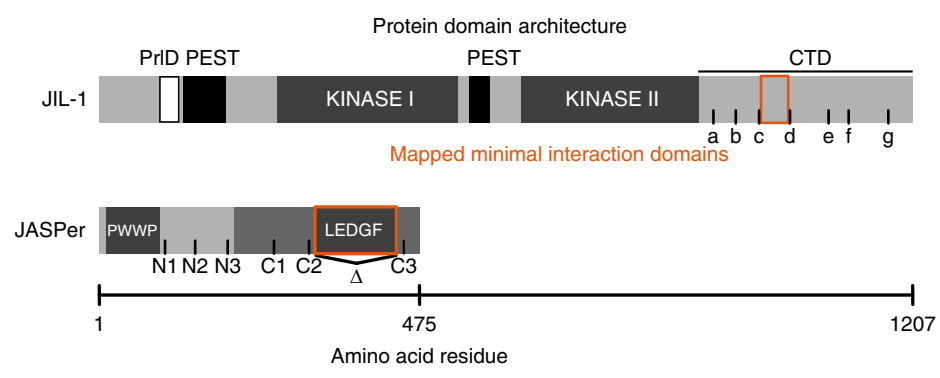

d

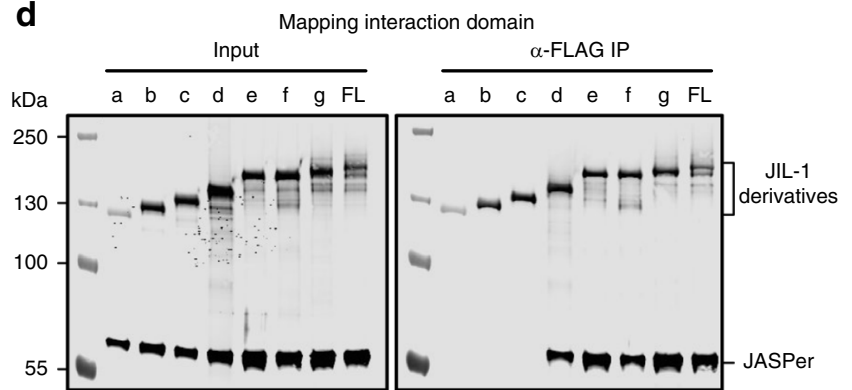

e

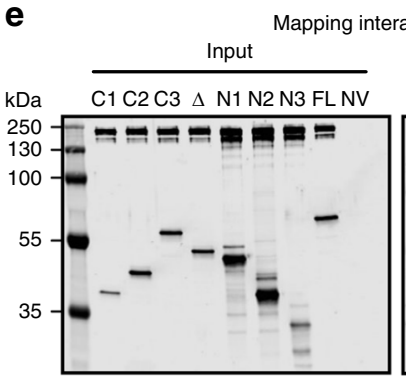

action domain

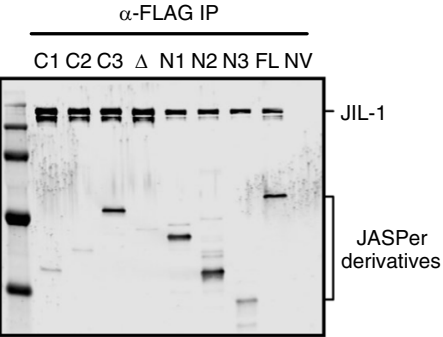

Fig. 1 JIL-1's C-terminal domain interacts with JASPer's LEDGF domain to form the JJ-complex. a Western blot analysis with $\alpha$-JASPer and $\alpha$-JIL-1 antibodies of co-IP from nuclear embryo extracts. Co-IP was performed with two different monoclonal $\alpha$-JASPer antibodies containing culture supernatants and culture medium as control. The corresponding unbound fractions are loaded next to each IP. Molecular weight markers are shown to the left. Source data are provided as a Source Data file. b SDS-PAGE with Coomassie staining of recombinant JJ-complex purification from Sf21 cells using a baculovirus dual expression system of FLAG-JIL-1 and untagged JASPer. Molecular weight markers are shown to the left. A contaminant band is marked by asterisk. Source data are provided as a Source Data file. c JIL-1 and JASPer domain architecture drawn to scale. In JIL-1, PEST domains are highlighted in black, kinase domains in dark gray and a predicted prion-like domain in white. In JASPer, PWWP and LEDGF domains are highlighted in dark gray and conserved region in intermediate gray. C-terminal truncation breakpoints a-g for JIL-1 and N-terminal (N1-N3) and C-terminal truncation breakpoints (C1-C3) for JASPer used in $\mathbf{d}$ and $\mathbf{e}$ are indicated. $\Delta$ denotes the deletion in JASPer- $\Delta$ LEDGF. $\mathbf{d}$ Western blot analysis using $\alpha-J I L-1$ and $\alpha$-JASPer antibodies of co-IP experiments with extracts from Sf21 cells expressing wild type, untagged JASPer and various FLAG-JIL-1 C-terminal deletion mutants. Co-IP was performed with $\alpha$-FLAG beads. Source data are provided as a Source Data file. e Western blot analysis as in d of co-IP experiments with extracts from Sf21 cells expressing various untagged JASPer deletion mutants and FLAG-JIL-1. Uninfected Sf21 cell extract was used as control (NV=no virus). Co-IP was performed with $\alpha$-FLAG beads. Source data are provided as a Source Data file.

JASPer stabilizes JIL-1 in vivo. To understand the function of JASPer in the JJ-complex, we generated JASPer ${ }^{c w 2}$ null allele by imprecise excision of the P-element in an appropriate EP line. The deletion encompassed the coding region of both described transcripts (Fig. 2a). Analysis of the salivary glands of homozygous JASPer ${ }^{\mathrm{Cw} 2 / \mathrm{cw}^{2}}$ mutants showed that JASPer was not detectable by western blot and on polytene chromosome spreads (Fig. 2b, c). Remarkably, JIL-1 was also not detectable in the absence of JASPer. The lack of the JJ-complex in turn correlates with undetected phosphorylation of H3S10, confirming that kinase as the major source of this modification in interphase (Fig. 2d). Such a direct relationship between kinase and H3S10ph cannot be seen in an exponentially growing cell population, due to the strong dominance of mitotic $\mathrm{H} 3 \mathrm{~S} 10 \mathrm{ph}^{6}$. As also described for the JIL-1 ${ }^{z 2 / z^{2}}$ hypomorph mutant ${ }^{14}$, global H3K9me2 levels were unchanged (Fig. 2b) but the mark redistributed from the chromocenter to the euchromatic chromosomal arms, in particular of the X chromosome (Fig. 2d). Although the JASPer ${ }^{\text {cw2 } / c w 2 ~}$ mutant mostly phenocopies JIL-1 $1^{z 2 / z 2}$ mutant, polytene chromosomes retain their characteristic banded pattern (Fig. 2c, d), which are lost in the $J I L-1^{z 2 / z 2}$ mutant ${ }^{30}$. This observation is consistent with the partial lethality of JASPer ${ }^{c w 2 / c w 2}$ mutant (54\% of expected survival, $n=1496$ ) as compared to the lethal $J I L-1^{z 2 / z 2}$ mutant (Fig. 2e). Ablation of JASPer by RNA interference in cultured cells also led to loss of JIL-1 (Fig. 2f). JIL-1 was depleted to the same level by RNAi against jil-1 or jasper in S2 and Kc cells, suggesting that JIL-1 is unstable in the absence of JASPer. The JIL-1 transcript level was unchanged upon jasper RNAi in our RNA-seq experiments, excluding regulation at the transcription level (Fig. 2g). However, trace amounts of JIL-1 or fragments of it might still be expressed and could explain the better viability of $J A S P e^{c w 2 / c w 2}$ mutant versus $J I L-1^{z 2 / z 2}$ mutant $^{31}$.

JASPer binds nucleic acids and H3K36me3 nucleosomes in vitro. In addition to the LEDGF JIL-1-binding domain, JASPer harbors a PWWP domain at its $\mathrm{N}$-terminus (Fig. 1c). PWWP domains have a positively charged surface favoring DNA binding and an aromatic pocket for methyl-lysine binding [for review ${ }^{32}$ ]. Conceivably, this domain is responsible for the recruitment of the JJ-complex to chromatin. As expected, recombinant JASPer had significant affinity for DNA in electrophoretic mobility shift assays (EMSA), while JIL-1 showed no detectable binding under the same conditions (Supplementary Fig. 4a). A 9-fold molar excess of JASPer shifted all DNA molecules. Apparently, several JASPer molecules can bind simultaneously one DNA molecule as at least three retarded bands appeared in the EMSA and the most retarded ones correlated with higher JASPer concentration. JASPer also bound a 123 nucleotide long RNA hairpin ${ }^{33}$ in a dose-dependent manner (Supplementary Fig. 4b). 
a

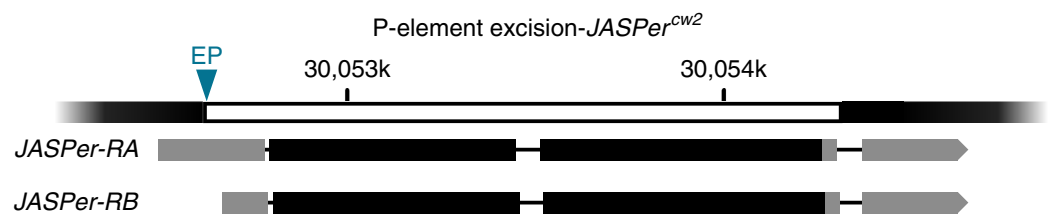

c

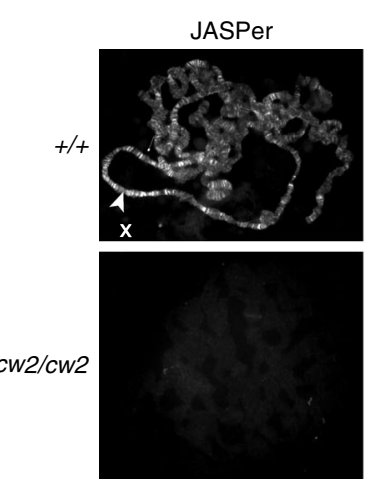

d
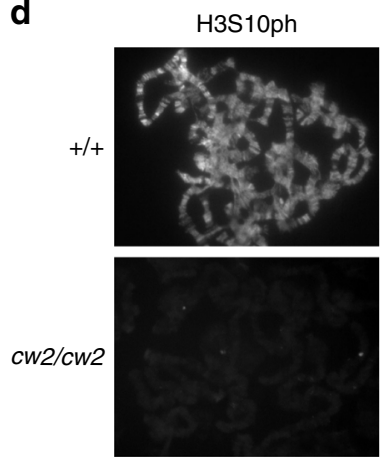

e

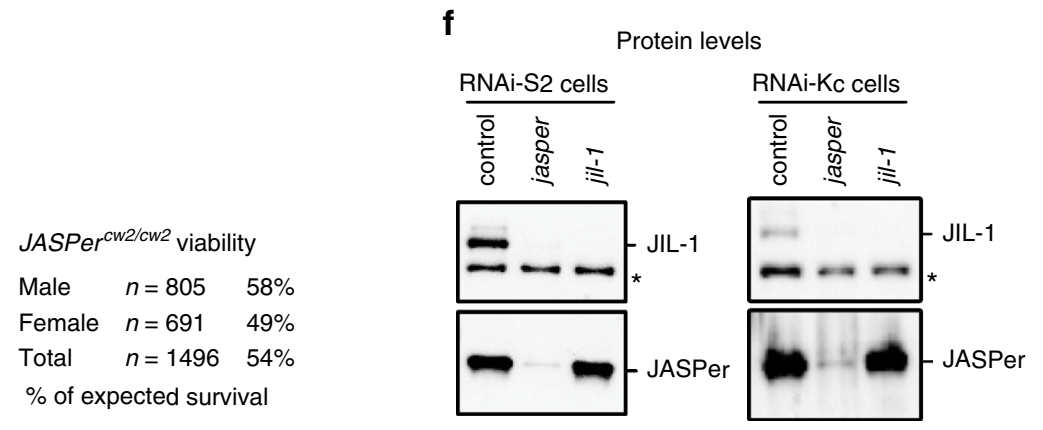

b
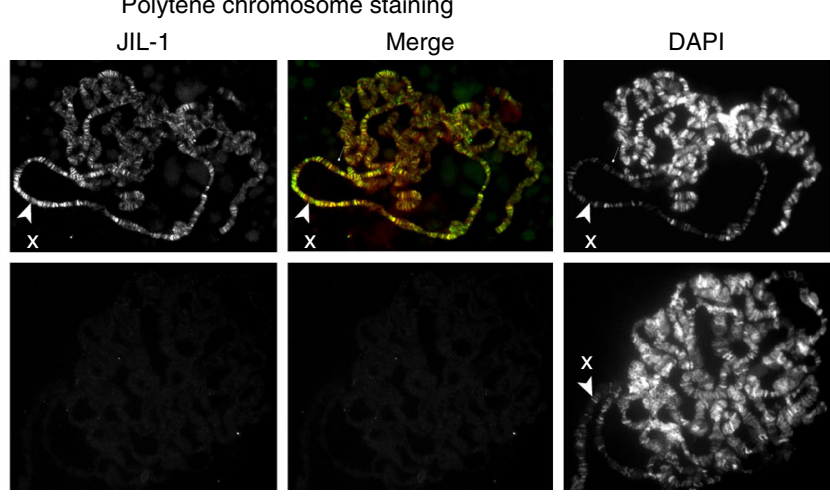

H3K9me2
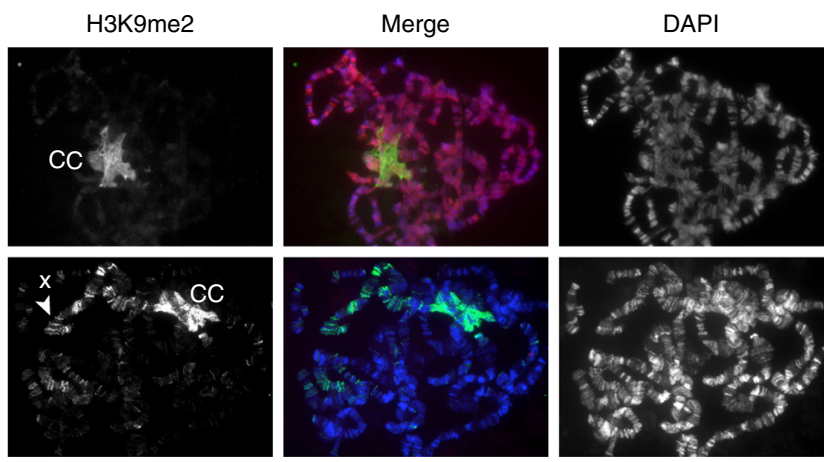
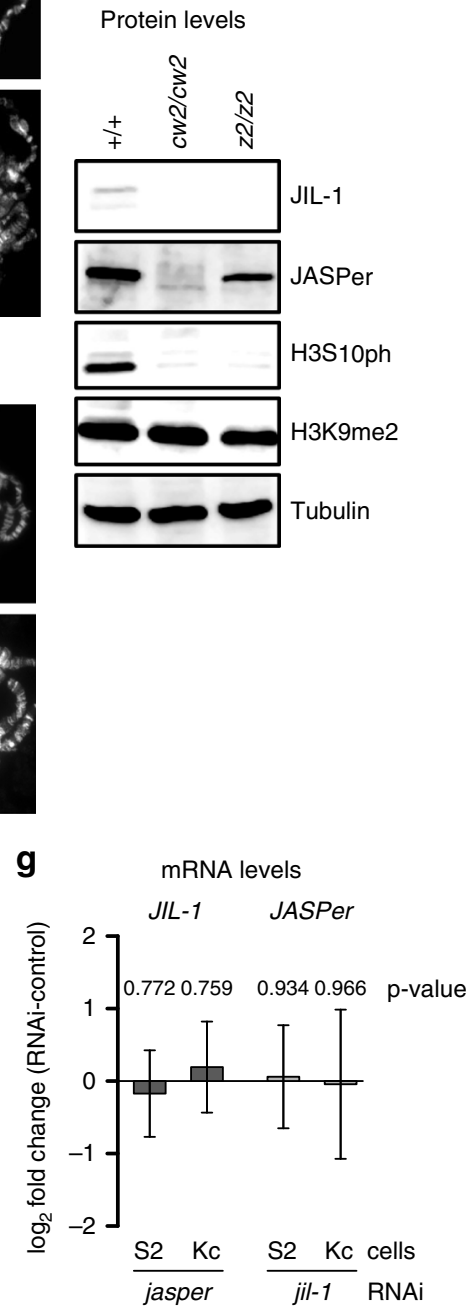

Fig. $2 \mathrm{JIL}-1$ is unstable in absence of JASPer in the JASPer ${ }^{\mathrm{cw} 2 / \mathrm{cw} 2}$ mutant and in cell lines. a Gene model for P-element excision in the JASPer locus to generate JASPer ${ }^{\mathrm{CW}} 2$ allele. The mRNA isoforms RA and RB are shown below. The excised genomic portion is marked in white and EP denotes the position of the excised P-element in EP-element line GS3268. b Western blot analysis of salivary gland extracts from L3 larvae of homozygous JASPercw2/cw2 and JIL$172 / z 2$ mutants and wild type larvae as control. Western blots using $\alpha-J I L-1, \alpha-J A S P e r, \alpha-H 3 S 10 p h$, and $\alpha-H 3 K 9 m e 2$ antibodies are shown, western blot with $\alpha$-tubulin antibody was used as loading control. c Immunofluorescence microscopy of polytene chromosome squashes from L3 larvae of homozygous JASPer rw2/cw2 and wild type larvae as control. From left to right, staining for JASPer, JIL-1, merged images and DNA are shown. The X chromosome is marked by arrow heads. Source data are provided as a Source Data file. d Immunofluorescence microscopy of polytene chromosome spreads from $L 3$ larvae of homozygous JASPercw2/cw2 and wild type larvae as control. From left to right, staining for H3S10ph, H3K9me2, merged images and DNA are shown. The $\mathrm{X}$ chromosome is marked by arrow head and the chromocenter is labeled with "CC". e Table summarizing viability of male and female JASPer ${ }^{\mathrm{CW} 2 / \mathrm{cw} 2}$ mutant flies. $\mathbf{f}$ Representative western blot analysis using $\alpha$-JASPer and $\alpha-J \mathrm{IL}-1$ antibodies on whole cell extracts from S2 cells (left panel) and Kc cells (right panel) after jasper or jil-1 RNAi treatment, as used for RNA-seq experiments. A cross-reacting band is marked by asterisk. Source data are provided as a Source Data file. $\mathbf{g}$ Bar chart showing mean $\log _{2}$ fold-change of normalized mean RNA-seq counts for JIL-1 and JASPer RNAi. Left panel, JIL-1 mRNA mean $\log _{2}$ fold-change upon jasper RNAi (S2 $n=4$ and Kc $\left.n=4\right)$. Right panel, JASPer mRNA mean $\log _{2}$ fold-change upon jil-1 RNAi (S2 $n=5$ and Kc $n=4$ ). Error bars represent standard error of the mean. 
a

Mononucleosome pull-down
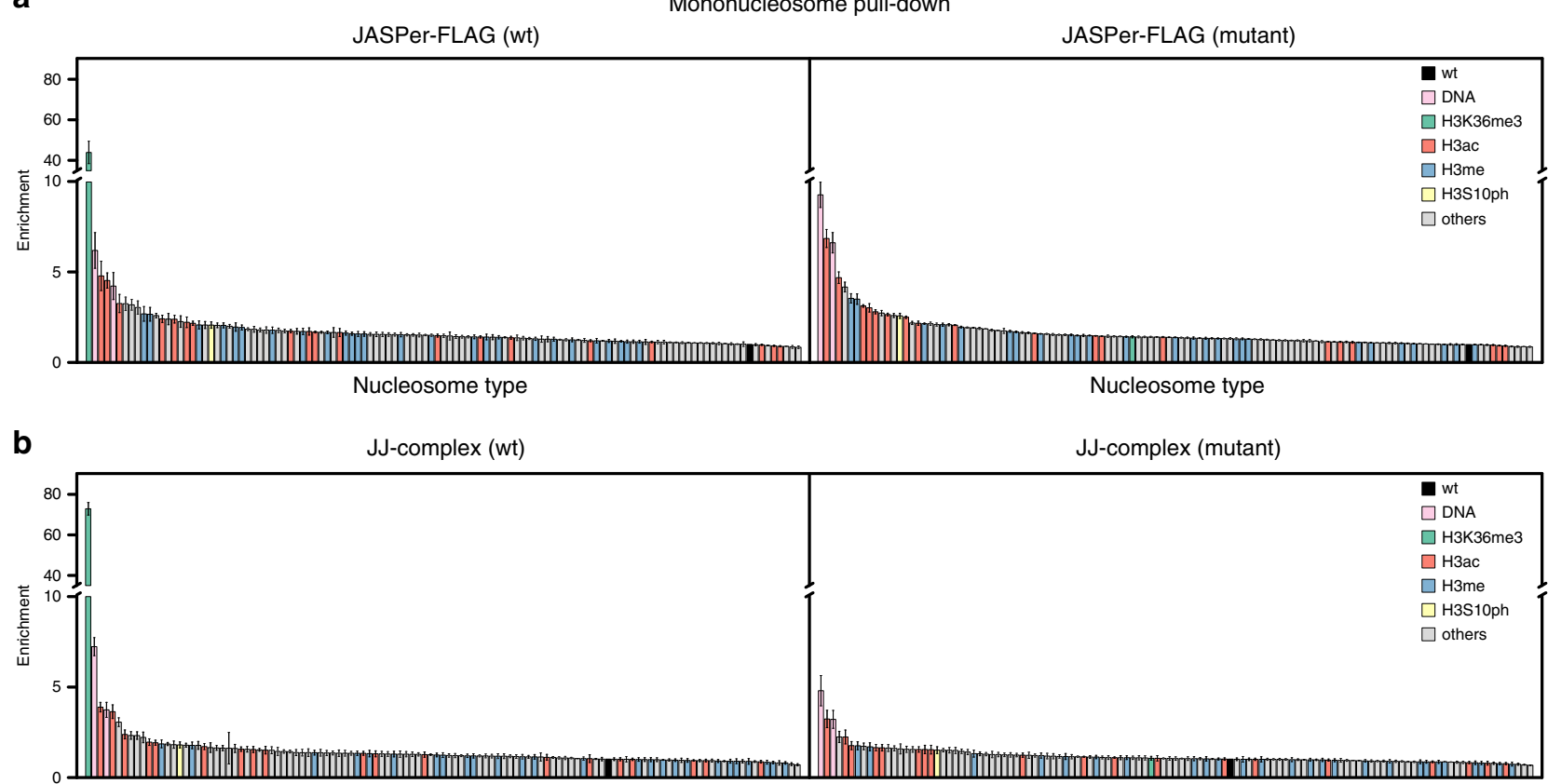

C

Chromosome 2R

ChIP-seq profiles - S2 cells

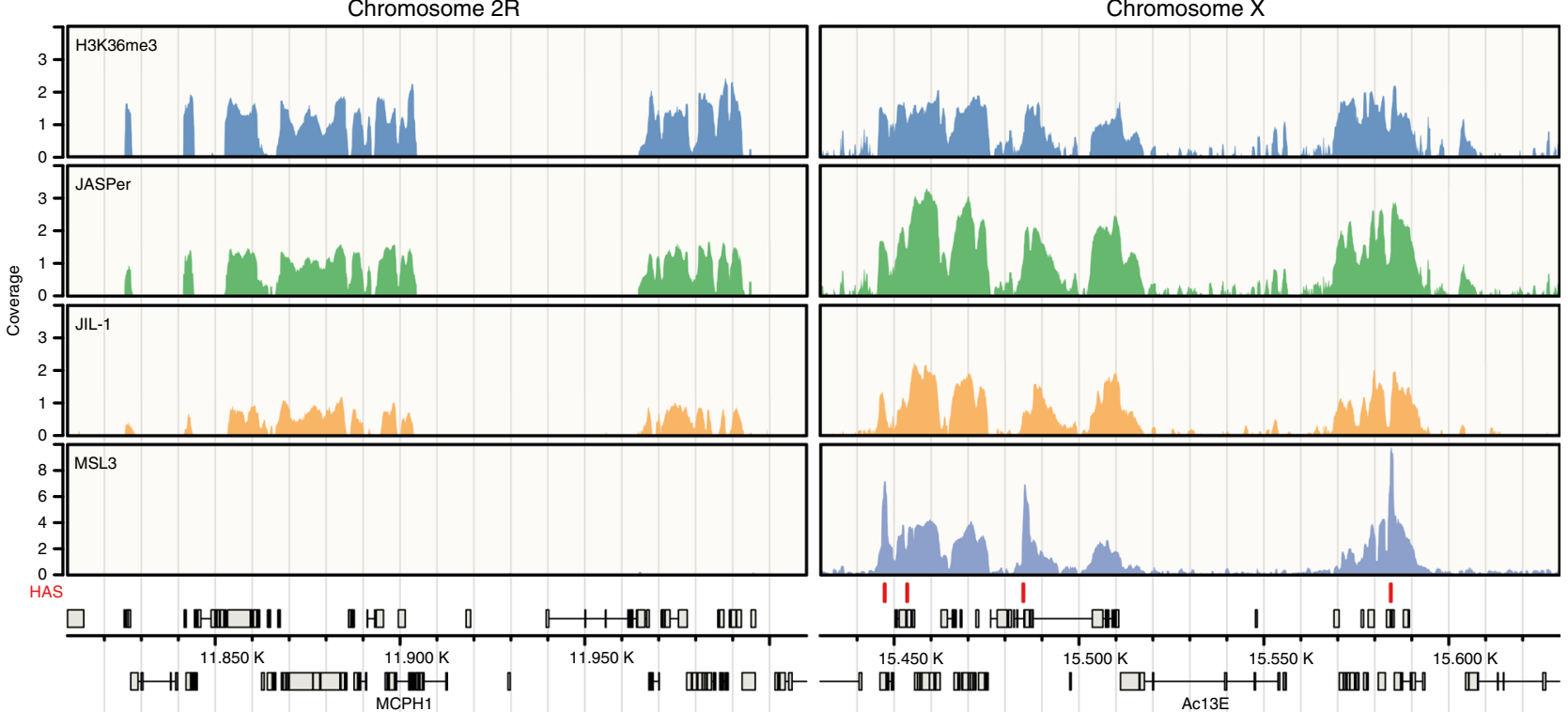

d

ChIP-seq coverage at genes-S2 cells
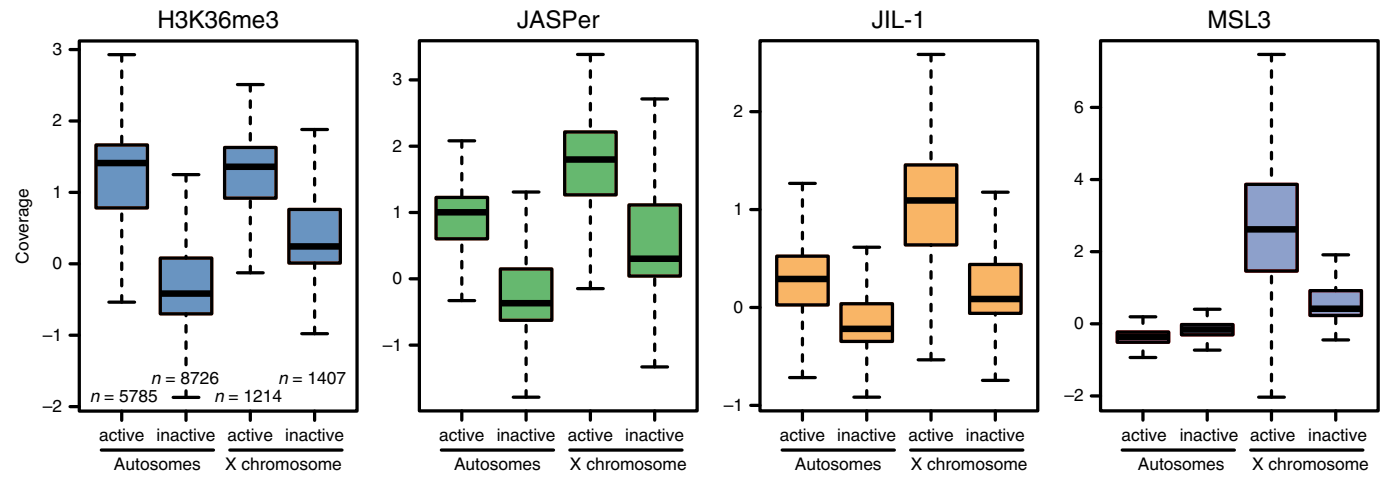
Fig. 3 The JJ-complex binds H3K36me3 nucleosomes in vitro and in vivo, and is enriched on the male X chromosome. a Bar chart of mean enrichment ( $n=$ 3 independent experiments with 2 different protein preparations) of nucleosome library pull-down with JASPer-FLAG (left panel) and aromatic cage mutant (right panel) relative to unmodified nucleosome, which is set to 1. Error bars represent standard error of the mean. $\mathbf{b}$ Bar chart of mean enrichment ( $n=3$ independent experiments) of nucleosome library pull-down with JJ-complex (FLAG-JIL-1 and untagged JASPer) (left panel) and aromatic cage mutant (right panel) relative to unmodified nucleosome, which is set to 1, as in a. Error bars represent standard error of the mean. $\mathbf{c}$ Genome browser profile showing mean H3K36me3 (upper panel, $n=4$ independent experiments), JASPer (second upper panel, $n=4$ independent experiments with 2 different antibodies), JIL-1 (second lower panel, $n=5$ independent experiments with 2 different antibodies) and MSL3 (lower panel, $n=3$ independent experiments) MNase ChIP-seq normalized coverage along representative $200 \mathrm{~kb}$ windows on chromosome $2 \mathrm{R}$ and X in male S2 cells. HAS are marked by red bars above the gene models in gray. $\mathbf{d}$ Box plot showing mean H3K36me3 (left panel, $n=4$ ), JASPer (second left panel, $n=4$ ), JIL-1 (second right, $n=$ 5) and MSL3 (right, $n=3)$ MNase ChIP-seq normalized coverage, as in $\mathbf{c}$, at active (tpm $>1)$ and inactive (tpm $\leq 1)$ genes on the autosomes $(n=5785$ and $n=8726$, respectively) and X chromosome ( $n=1214$ and $n=1407$, respectively) in male S2 cells. Box plot elements are defined as center line marking the median, box limits are the upper and lower quartiles, whiskers extend maximally 1.5-times the interquartile range and outliers are removed.

To decipher the binding specificity of JASPer and the JJcomplex for nucleosomes, we used a library of 115 different types of DNA-barcoded nucleosomes bearing different histone and DNA modifications ${ }^{34}$. Recombinant, FLAG-tagged JASPer or FLAG-tagged JJ-complex were coupled to a-FLAG beads, incubated with the nucleosome library, washed and the pulleddown nucleosomes were quantified by sequencing of the associated indexes. Wild type JASPer showed high specificity towards nucleosomes bearing the single $\mathrm{H} 3 \mathrm{~K} 36 \mathrm{me} 3$ modification (Fig. 3a). This modification was $\sim 40$-fold enriched in the IP relative to the unmodified nucleosome used for normalization. Mutation of two residues in the aromatic cage to alanines (Y23A and W26A) abolished specific H3K36me3 binding (Fig. 3a). Similar results were obtained for the JJ-complex, where H3K36me3 was 70-fold enriched over the unmodified nucleosome, only if the aromatic cage is intact (Fig. 3b). In accordance with the DNA binding activity shown in EMSA (Supplementary Fig. 4a), we found a $\sim 3$-fold to 9-fold enrichment of the two nucleosome-free DNAs used as controls in the library. The enrichment ( 2-fold to 7-fold) of nucleosomes bearing acetylated $\mathrm{H} 3$ tails, could be due to their lower assembly efficiencies, as described $^{34}$. Alternatively, it could reflect, at least in part, the better accessibility of the linker DNA in those nucleosomes, as acetylation of the $\mathrm{H} 3$ tail decreases its binding to the linker $\mathrm{DNA}^{35-37}$. Acetylation may thus favor linker DNA-dependent binding by JASPer, as shown for the PWWP domain of PWWP2 $\mathrm{A}^{35}$. These results point towards a mostly ionic interaction between the overall positively charged JASPer ( $\mathrm{pI}$ of 8.3) and the negatively charged sugar-phosphate backbone of the DNA as has been proposed for other PWWP domains to synergize with the aromatic cage for high-affinity binding of H3K36me3 nucleosomes. The PWWP domain contacts both DNA gyres next to the $\mathrm{H} 3$ tail exit site through its basic surface and the aromatic cage engages with the K36me3 residue ${ }^{38,39}$.

JIL-1 is a potent kinase in vitro and phosphorylates isolated $\mathrm{H} 3$ peptide (amino acids 1-20) and full-length histone H3. However, the isolated kinase proved to be inactive on nucleosome arrays in vitro even at high molar ratios of kinase to nucleosome ${ }^{6}$. To explore whether the oriented binding of the JJ-complex to nucleosomes would favor phosphorylation, we used a semiquantitative kinase assay based on western blot detection of H3S10ph. Using H3K36me3-modified and unmodified nucleosomes and 12-mer nucleosome arrays (Supplementary Fig. 4c,d), we confirmed that only the wild type JJ-complex phosphorylates $\mathrm{H} 3 \mathrm{~S} 10$, that the active site-mutated enzyme is inactive and that we could detect low amounts of H3S10ph by western blot (Supplementary Fig. 4e). For the kinase assay with nucleosomes, we had to load $\sim 10$-times more of each reaction to detect similar levels of H3S10ph as compared to a completely phosphorylated, isolated $\mathrm{H} 3$, indicating that the JJ-complex is $\sim 10$-times less active on nucleosomes (Supplementary Fig. 4f). However, our analysis showed that altogether JIL-1 in the JJ-complex is more active on nucleosome arrays ( $>3 \%$ of the phosphorylated $\mathrm{H} 3$ reference) than on mononucleosomes suggesting that the binding to one nucleosome in the array may facilitate the phosphorylation of a neighboring nucleosome (Supplementary Fig. 4f,g). The fact that we did not observe a preference for $\mathrm{H} 3 \mathrm{~K} 36 \mathrm{me} 3$ nucleosomes is probably due to the high concentration of JJ-complex used in the kinase assay to allow H3S10ph detection by western blot.

The JJ-complex localizes to active chromatin in vivo. Because the JJ-complex specifically selects $\mathrm{H} 3 \mathrm{~K} 36 \mathrm{me} 3$ nucleosomes via the PWWP of JASPer in vitro, we wished to confirm this interaction in vivo. Recently, differing results about JIL-1 localization in vivo arose from data generated using different ChIP-chip/-seq approaches $6,8,21$, possibly due to different chromatin fragmentation protocols ${ }^{40}$. To clarify this issue, we used both chromatin digested with MNase and chromatin sheared by sonication for ChIPs of H3K36me3, JIL-1, JASPer, and MSL3 in male S2 cells and in female Kc cells (Supplementary Fig. 5a-c). Independent of the fragmentation strategy, we found that JIL-1 and JASPer binding profiles overlap with $\mathrm{H} 3 \mathrm{~K} 36 \mathrm{me} 3$ at exons of active genes in vivo (Fig. 3c, Supplementary Fig. 6a-d), as expected ${ }^{41}$. Like JIL1 , JASPer is enriched at active genes on the $\mathrm{X}$ chromosome relative to autosomes only in male S2 cells (Fig. 3d, Supplementary Fig. 7a,b). We excluded that this enrichment is caused by normalization due to copy number differences by comparing the non-input-normalized coverages of $\mathrm{H} 3 \mathrm{~K} 36 \mathrm{me} 3$, JASPer, and JIL1 to the input (Supplementary Fig. 7c-e). In female Kc cells, we found similar coverages of $\mathrm{H} 3 \mathrm{~K} 36 \mathrm{me} 3$ and JASPer at active genes on all chromosomes, whereas in male $\mathrm{S} 2$ cells the $\mathrm{X}$ chromosomal sequence coverage of $\mathrm{H} 3 \mathrm{~K} 36 \mathrm{me} 3$ is roughly half of the autosomal one, as for the input. By contrast, coverages of JASPer and JIL-1 on active $\mathrm{X}$ chromosomal and autosomal genes in male S2 cells are similar. Interestingly, the X-chromosomal enrichment of JASPer and JIL-1 is only observed in male cells (Fig. 3d and Supplementary Fig. 7a).

The active genes on the $\mathrm{X}$ chromosome in male cells are strongly acetylated at $\mathrm{H} 4 \mathrm{~K} 16$ by the DCC subunit MOF, which is thought to decompact the chromatin fiber ${ }^{42,43}$. This loosening of chromatin folding may allow JASPer to bind better to H3K36me3, independent of JIL-1. To test whether the Xchromosomal enrichment of JASPer depends on JIL-1, we analyzed JASPer, MSL3, H4K16ac, and H3K9me2 distribution by ChIP-seq after jil-1 RNAi in S2 cells (Fig. 4a and Supplementary Fig. 5d). To quantify the absolute difference in ChIP-seq coverage between conditions by spike-in normalization, we added $5 \% D$. virilis cells to our chromatin preparations ${ }^{44}$. Intriguingly, the X chromosome-specific enrichment of JASPer in male S2 cells was reduced to the autosomal level in absence of JIL1, while the DCC subunit MSL3 was slightly redistributed, the diagnostic H4K16ac, set by the DCC, slightly dropped and the 
a ChIP-seq profiles-S2 cells

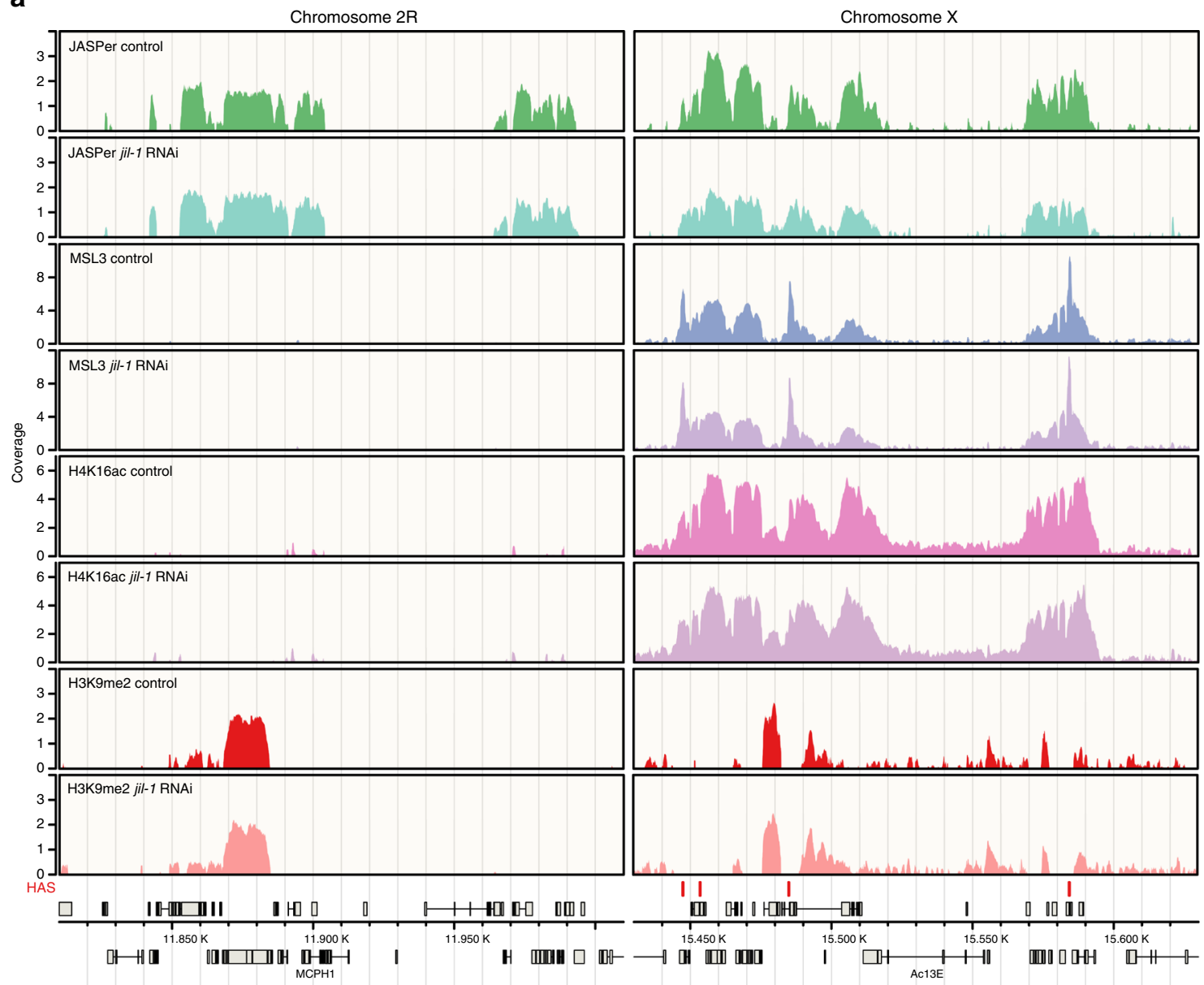

b

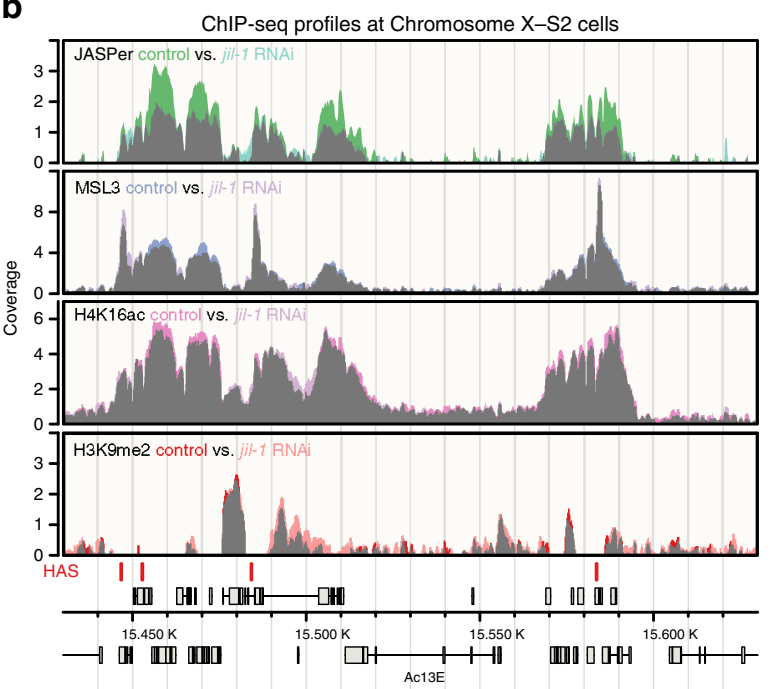

C

ChIP-seq coverage at active genes-S2 cells
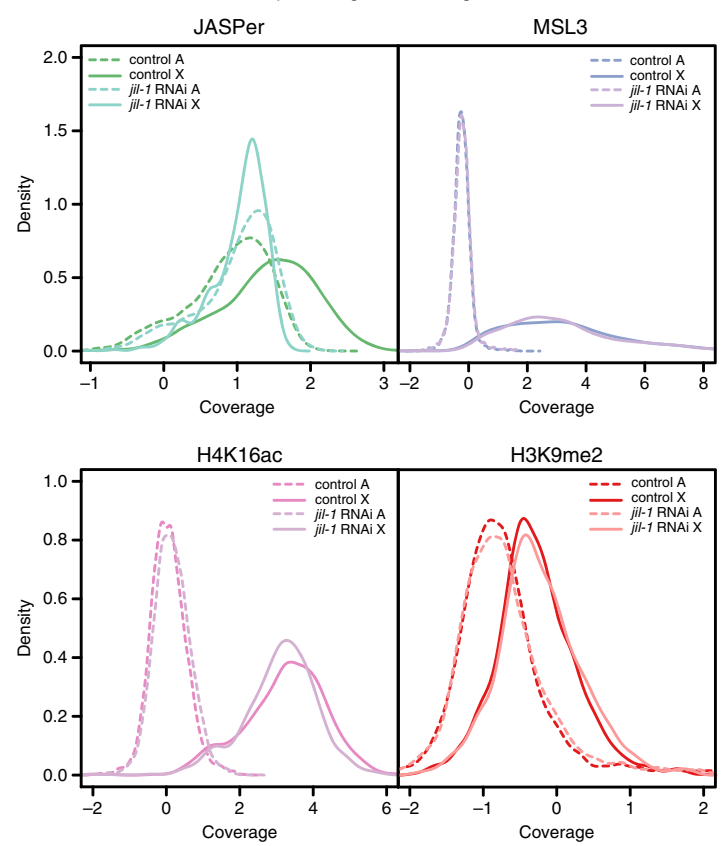

heterochromatin mark H3K9me2 slightly increased (Fig. 4 and Supplementary Fig. 8a,b). This demonstrates that JASPer per se does not need JIL-1 for H3K36me3 interaction, but its binding is enhanced on the male $\mathrm{X}$ chromosome in the JJ-complex. Interestingly, the loss of JASPer after depletion of JIL-1 is stronger closer to the $\sim 300$ high affinity sites (HAS) bound by the
DCC along the X chromosome (Supplementary Fig. 8c). Concomitantly, the spreading of MSL3 from HAS is slightly diminished and the H4K16ac density slightly drops but independently of the distance to HAS after jil-1 RNAi. These small differences in the dosage compensation hallmark probably cannot explain the loss of JASPer enrichment. It thus appears that 
Fig. 4 JIL-1 and not H4K16ac is responsible for the enrichment of JASPer at the male X chromosome. a Genome browser profile showing mean ( $n=3$, for MSL3 $n=2$ ) spike-in ChIP-seq normalized coverage in control male S2 cells and after jil-1 RNAi treatment from top to bottom for JASPer, MSL3, H4K16ac and $\mathrm{H} 3 \mathrm{~K} 9 \mathrm{me} 2$ along representative $200 \mathrm{~kb}$ windows on chromosome $2 \mathrm{R}$ and X. HAS are marked by red bars above the gene models in gray. $\mathbf{b}$ Genome browser profile as in a showing mean $(n=3$, for MSL3 $n=2)$ spike-in ChIP-seq normalized coverage in control male S2 cells and after jil- 1 RNAi treatment, from top to bottom for JASPer, MSL3, H4K16ac, and H3K9me2 along a representative $200 \mathrm{~kb}$ window on chromosome X. Signal overlay is marked in grey. c Density plot showing mean $(n=3$, for MSL3 $n=2)$ spike-in ChIP-seq normalized coverage in control male S2 cells and after jil-1 RNAi treatment at active (tpm >1) genes for JASPer (top left), MSL3 (top right), H4K16ac (bottom left), and H3K9me2 (bottom right). X chromosomal genes ( $n=1214)$ are represented by a solid line and autosomal genes (chromosomes $2 \mathrm{~L}, 2 \mathrm{R}, 3 \mathrm{~L}$, and $3 \mathrm{R}, n=5785$ ) by a dashed line.

JASPer's enrichment on the male $\mathrm{X}$ chromosome depends directly on JIL-1.

Because the main difference between the $\mathrm{X}$ chromosome and autosomes is the presence of the DCC and gene-body H4K16 acetylation, the enrichment of the JJ-complex on the $\mathrm{X}$ chromosome may be due to functional interactions of the JJcomplex with the DCC. Direct interaction of JIL-1 with MSL1 and MSL3 subunits of the DCC had been shown in vitro ${ }^{45}$, but so far no clear direct association of the two endogenous complexes has been documented (see also below). We explored the interaction between the two recombinant complexes after expression from baculovirus vectors. Extracts containing JJcomplex (FLAG-JIL-1/untagged JASPer) on the one hand and a partial DCC consisting of MSL1, MSL2, and MSL3 (FLAGMSL1/untagged MSL2/FLAG-MSL3) on the other hand were mixed in appropriate stoichiometry ${ }^{46}$ and specific antibodies were used for IP (Supplementary Fig. 8d). The MSL1 antibody retrieved not only the associated MSL2 and MSL3, but also some JJ-complex. Conversely, the JIL-1 antibody immunoprecipitated MSL proteins in addition to abundant JJ-complex. This suggests that the two complexes may directly interact with each other. Altogether, the enrichment of the JJ-complex on the male $\mathrm{X}$ chromosome may be explained, at least in part, by a JIL-1-dependent interaction between the JJ-complex and the DCC.

The JJ-complex supports expression of male X-linked genes. As we confirmed that the JJ-complex binds to active gene bodies, we wished to explore the functional consequences. To do so, we quantified the transcriptome changes by RNA-seq after RNAi depletion of JASPer or JIL-1 in male S2 and female Kc cells. PCA analysis showed that jasper and jil-1 RNAi affected overall gene expression similarly (Supplementary Fig. 9a). The per-gene analysis showed that upon jasper and jil-1 RNAi in both cell lines the transcription of many genes changed over a wide range of expression levels, with more genes being downregulated $(\mathrm{fdr}<$ $0.05)$ (Fig. 5a). The changes upon jasper and jil-1 RNAi correlate ( $r=0.597$ in S2 and $r=0.561$ in Kc cells), indicating that depletion of the JJ-complex and of JIL-1 alone result in a similar phenotype (Supplementary Fig. 9b). Remarkably, transcription of $\mathrm{X}$ chromosomal genes is globally reduced upon depletion of either protein in male S2, but not in female Kc cells (Fig. 5b). We showed earlier that mapping JIL-1-dependent interphase $\mathrm{H} 3 \mathrm{~S} 10 \mathrm{ph}$ in exponentially growing cells is not possible due to the overwhelming levels of mitotic $\mathrm{H} 3 \mathrm{~S} 10 \mathrm{ph}^{6}$. Instead, we monitored changes in the diagnostic histone modifications $\mathrm{H} 3 \mathrm{~K} 9 \mathrm{me} 2$ for heterochromatin and $\mathrm{H} 4 \mathrm{~K} 16 \mathrm{ac}$ for dosage compensation upon JIL-1 depletion in male S2 cells using the spike-in ChIP-seq approach (Supplementary Fig. 9c). In agreement with a decreased expression of X-chromosomal genes, a small increase of $\mathrm{H} 3 \mathrm{~K} 9 \mathrm{me} 2$ and a slight decrease of H4K16ac were observed. In a gene-by-gene analysis of X-linked genes, we observed that downregulated genes consistently tend to lose H4K16ac and JASPer or gain H3K9me2 (Supplementary Fig. 10). We related the slight increase of $\mathrm{H} 3 \mathrm{~K} 9 \mathrm{me} 2$ to an increased susceptibility of the $\mathrm{X}$ chromosome to invasion of patches of heterochromatin as also seen in control cells (Supplementary Fig. 9c). The decrease in $\mathrm{H} 4 \mathrm{~K} 16 \mathrm{ac}$ on the male X chromosome observed both globally (Fig. 4a,b) and specifically at expressed genes (Supplementary Fig. 9c) suggests that JIL-1 may affect H4K16ac indirectly.

Altogether our results suggest that JIL-1 overall positively regulates gene expression and that the effect is most pronounced on the $\mathrm{X}$ chromosome in male cells.

The JJ-complex regulates expression of telomeric transposons. JIL-1 is the only known activator of the expression of non-LTR retrotransposons of the HTT arrays (HeT-A, TAHRE, and TART$A / B / C)$, which is essential for telomere maintenance in Drosophila ${ }^{18,19}$. Mapping our ChIP-seq data to the consensus sequences of $126 \mathrm{D}$. melanogaster transposable elements (TEs) we found that a subset of them showed an enrichment of $\mathrm{H} 3 \mathrm{~K} 36 \mathrm{me} 3$ and JJ-complex in S2 cells (Fig. 6a). H3K36me3, JASPer, and JIL1 are strongly enriched at all transposons of the HTT arrays, as well as at the LTR-retrotransposons Gypsy5 and 3S18 (Fig. 6a). Depletion of JIL-1 and JASPer by RNAi led to statistically significant reduced expression $(\mathrm{fdr}<0.05)$ of the majority of TEs (Fig. 6b, Supplementary Fig. 11a). The good correlation of the effects of each RNAi $(r=0.850)$ supports the idea of a joint action of JIL-1 and JASPer in a functional complex (Supplementary Fig. 11b). Among telomeric TEs, which are bound by the JJcomplex, the expression of $H e T-A$ and TART- $A$ is reduced after JASPer depletion and TART-B and TART-C are additionally downregulated after JIL-1 depletion. However, we do not robustly detect expression of TAHRE. Even though we found many more significantly downregulated TEs in S2 cells, we propose that this is indirect as these TEs lack detectable H3K36me3 enrichment and JJ-complex binding (Fig. 6a, b). However, the TEs of the HTT arrays seem to be mostly active and lack H3K9me2. Upon JIL-1 depletion, we detected an increase in $\mathrm{H} 3 \mathrm{~K} 9 \mathrm{me} 2$ at the TEs of the HTT arrays, except for TART-C (Fig. 6c, Supplementary Fig. 11). Concomitantly, the enrichment of JASPer is decreased at all transposons of the HTT arrays upon JIL-1 depletion (Supplementary Fig. 12), suggesting that either H3K36me3 is decreased there because of the lower expression and/or JIL-1 contributes to the enrichment of the JJ-complex at telomeres.

Altogether, we propose that TE's of the HTT arrays acquire $\mathrm{H} 3 \mathrm{~K} 36 \mathrm{me} 3$ when they are transcribed and recruit the JJ-complex to maintain their active state at least in part by preventing heterochromatization.

The JJ-complex associates with other chromatin complexes. To elucidate the interaction network of the JJ-complex, we immonoprecipitated JASPer with various antibodies under stringent conditions from embryo extracts and identified associated proteins by mass spectrometry. We identified 69 statistical significantly enriched proteins ( $p$-value $<0.05$ and $\log _{2}$ fold-change >4) (Fig. 7a, Supplementary Data 1). The five most enriched GO terms associated to those proteins include 'chromatin remodeling', 'protein acetylation', 'chromatin organization', and 

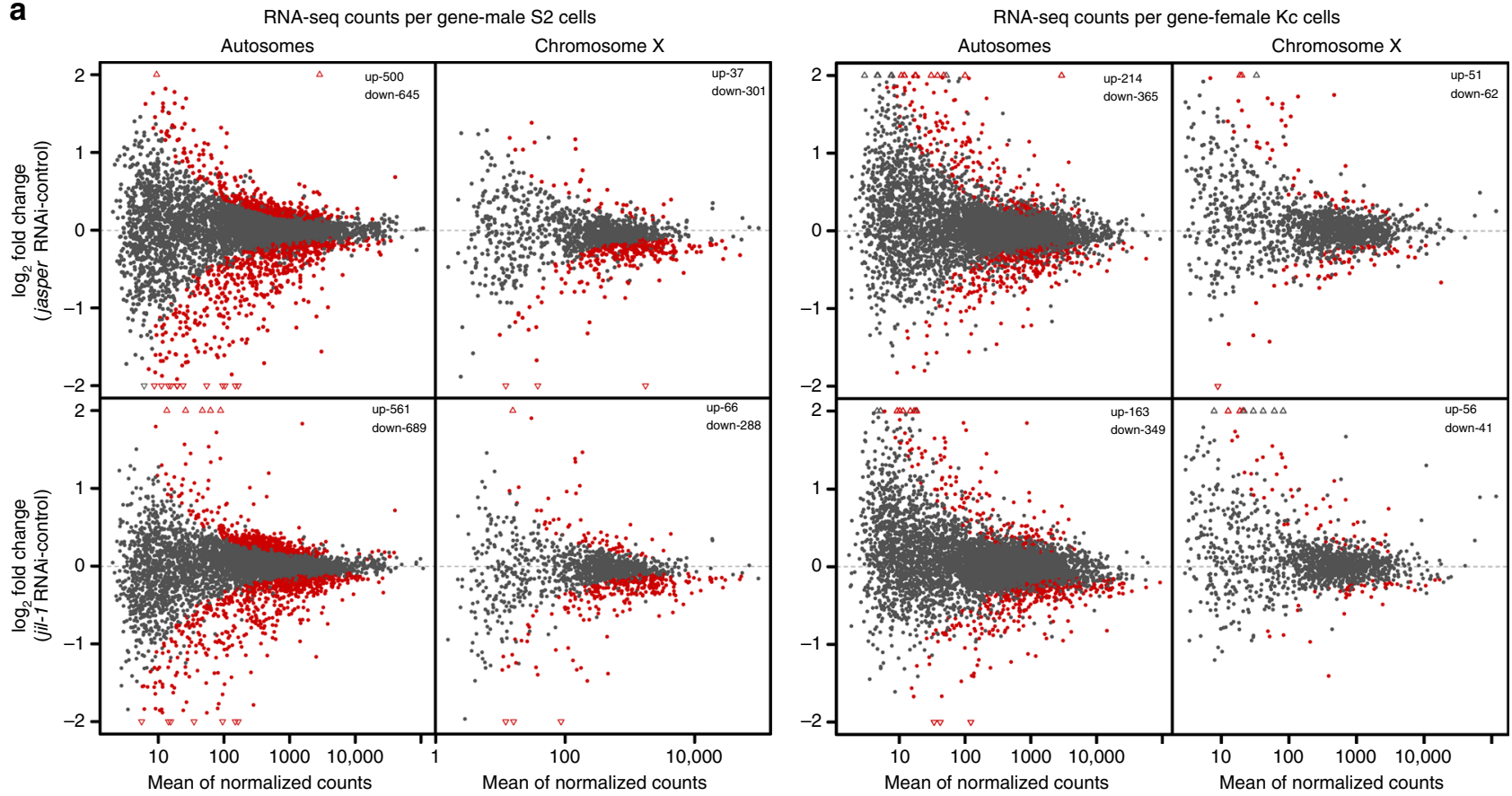

b
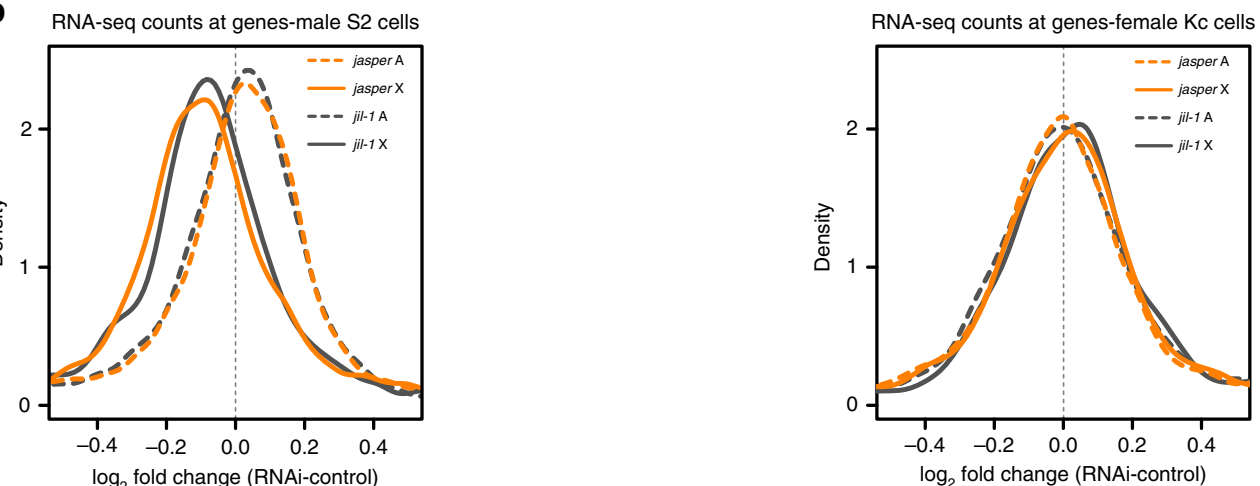

Fig. 5 JIL-1 and JASPer depletion in cells modulates the transcriptional output of genes, especially on the male $X$ chromosome. a MA-plot showing mean $\log _{2}$ fold-change of RNA-seq counts upon jasper RNAi versus control (upper panel, $n=4$ ) and jil-1 RNAi versus controls (lower panel, $n=5$ ) against mean RNA-seq counts for robustly detected genes at autosomes (left, chromosomes $2 \mathrm{~L}, 2 \mathrm{R}, 3 \mathrm{~L}$, and $3 \mathrm{R} n=6833$ ) and $\mathrm{X}$ chromosome (right, $n=1441$ ) in male S2 cells (left site). Statistically significant differentially expressed genes between RNAi and control conditions (fdr $<0.05)$ are marked in red and the number of significant genes is indicated on the plot. On the right, mean $\log _{2}$ fold-change of RNA-seq counts upon jasper RNAi versus control (upper panel, $n=4$ ) and jil-1 RNAi versus controls (lower panel, $n=4$ ) against mean RNA-seq counts for autosomal genes (left, chromosomes $2 \mathrm{~L}, 2 \mathrm{R}, 3 \mathrm{~L}$, and $3 \mathrm{R} n=$ 7144) and $X$ chromosomal genes (right, $n=1509$ ) in female Kc cells (left site). b Density plot showing mean log $\operatorname{lol}_{2}$ fold-change of RNA-seq counts upon jasper RNAi versus controls $(n=4)$ and jil-1 RNAi versus controls $(n=5)$ at genes in male S2 cells, in left panel, as in a. X chromosomal genes $(n=1441)$ are marked with solid line and autosomal genes (chromosomes $2 \mathrm{~L}, 2 \mathrm{R}, 3 \mathrm{~L}$, and $3 \mathrm{R}, n=6833$ ) with dashed line and jasper RNAi additionally in orange. Right panel, mean $\log _{2}$ fold-change of RNA-seq counts upon jasper RNAi and jil-1 RNAi versus controls ( $n=4$ each) at genes in female Kc cells. X chromosomal genes $(n=1509)$ and autosomal genes (chromosomes $2 \mathrm{~L}, 2 \mathrm{R}, 3 \mathrm{~L}$, and $3 \mathrm{R}, n=7144)$.

'transcription from RNA Pol II promoters' and its regulation (Fig. 7b). Among the most enriched interacting proteins we found BOD1, Dpy-30L1, Rbbp5, and Set1, subunits of the Set1/ COMPASS complex mediating promoter-proximal $\mathrm{H} 3 \mathrm{~K} 4$ dimethylation and trimethylation [for review ${ }^{47}$. Dpy-30L1 and Rbbp5 are common subunits of the different COMPASS complexes, containing one of the three histone methyltransferases Set1, Trx, and Trl in flies. Interestingly, BOD1/CG5514 had not been described in the D. melanogaster Set1/COMPASS complex but is a specific subunit of the Set1B/COMPASS complex in humans 48,49 . The next most represented interactors were the related PBAP and Brm remodeling complexes with e(y)3, polybromo, Bap170, Bap111, and Snr1 (Fig. 7a and Supplementary Fig. 13). Further subunits of the PBAP/Brm complex and other subunits of remodeling complexes were also enriched, though below statistical significance of this experiment (Fig. 7b). Furthermore, we found the heterochromatin components Su(var)3-7 and $\mathrm{Su}(\mathrm{var}) 205$ (HP1) significantly enriched (Fig. 7a), which are known to genetically interact with JIL-1 ${ }^{14,15}$. Several published interactors of JIL-1, like Chromator ${ }^{50}$ or MSL1 and MSL ${ }^{45}$ were not detected or not significantly enriched, possibly because of more dynamic association. Among the subunits of the DCC, only MOF was detected together with other subunits of the alternative MOF-containing NSL (non-specific-lethal) complex (Fig. 7a). NDF (nucleosome destabilizing factor) which was found associated with JIL- 1 by mass spectrometry after cross-linking ${ }^{51}$ was also enriched (Fig. 7a). NDF has recently been shown to destabilize nucleosomes in front of the transcribing polymerase, but 
a

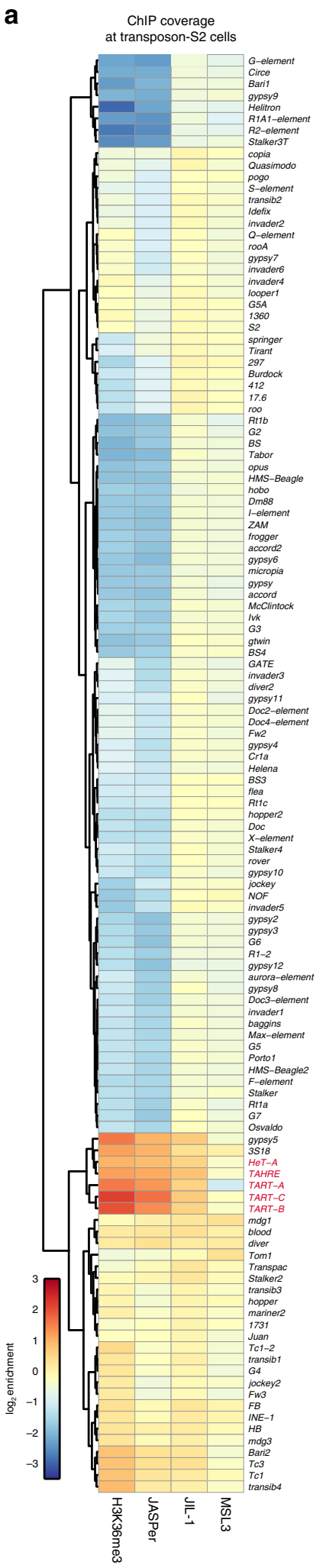

b
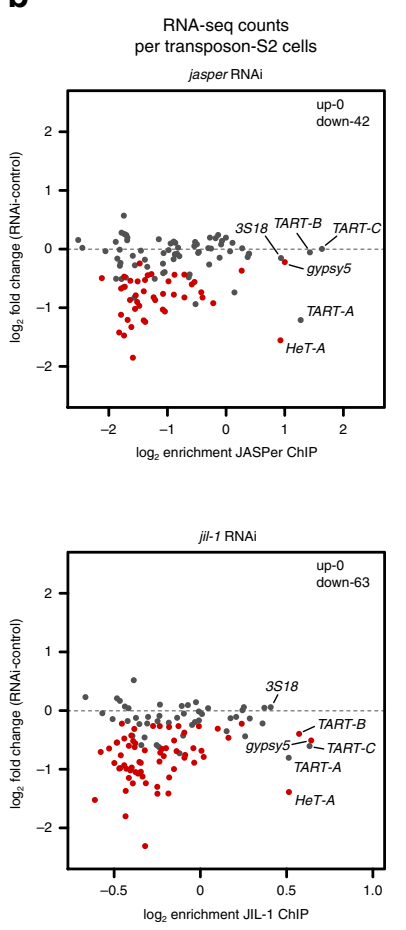

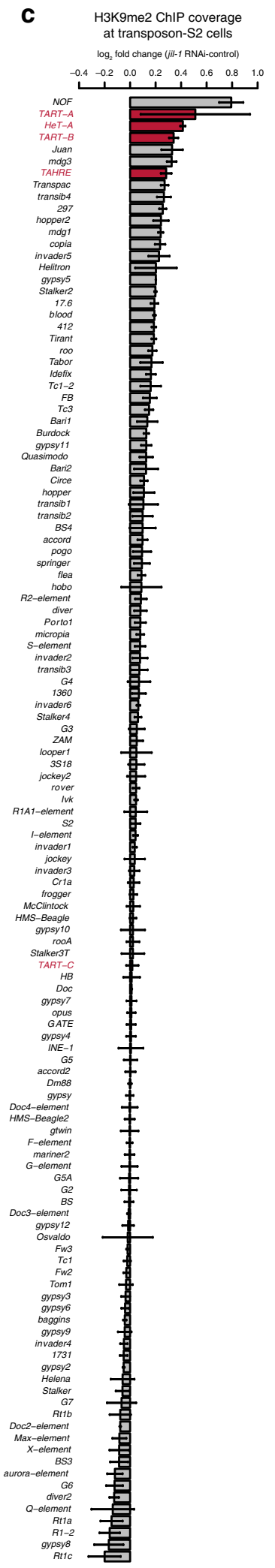

its depletion had only minor effects on overall transcript levels ${ }^{52}$. We speculated that the JJ-complex and NDF may have redundant functions on transcription. Therefore, we compared the transcriptome changes in male S2 cells after RNAi depletion of either JASPer or NDF alone, or in combination. Although, the depletion efficiency is only partial for NDF (Supplementary Fig. 13a), PCA separates the single jasper and $n d f$ RNAi samples well from the control samples (Supplementary Fig. 13b). However, the combined depletions showed no increased variance, and the expression changes after JASPer or NDF depletion show only a weak correlation $(r=0.39$, Supplementary Fig. 13c). Although, JASPer and NDF co-localize to active genes marked by H3K36me3, they seem to not have redundant roles in the regulation of steady state mRNA levels. 
Fig. 6 JIL-1 and JASPer depletion in S2 cells decrease the transcript level of transposons of the telomeric transposons of the HTT arrays. a Heatmap showing mean normalized $\log _{2}$ enrichment in H3K36me3 $(n=4)$, JASPer $(n=4)$, JIL-1 $(n=5)$, and MSL3 $(n=3)$ MNase ChIP-seq at transposons $(n=$ 124) in male S2 cells. Transposons of the HTT array are marked in red. b Scatter plot showing mean $\log _{2}$ fold-change of RNA-seq counts upon jasper RNAi versus control and jil-1 RNAi versus control against mean normalized $\log _{2}$ enrichment in JASPer $(n=4$, upper panel) and JIL-1 ( $n=5$, lower panel) MNase ChIP-seq, respectively, at robustly detected transposons in male S2 cells $(n=111)$. Statistically significant differentially expressed transposons between RNAi and control conditions ( $\mathrm{fdr}<0.05)$ are marked in red and the number of significant genes is indicated on the plot. TEs of the HTT arrays, gypsy5, and $3 S 18$ are labeled. c Bar plot of difference of mean H3K9me2 $(n=3$ each) spike-in ChIP-seq normalized coverage after jil-1 RNAi treatment and control male S2 cells at transposons $(n=124)$ in male S2 cells. Error bars represent standard error of the mean. TEs of the HTT arrays are marked in red.

a

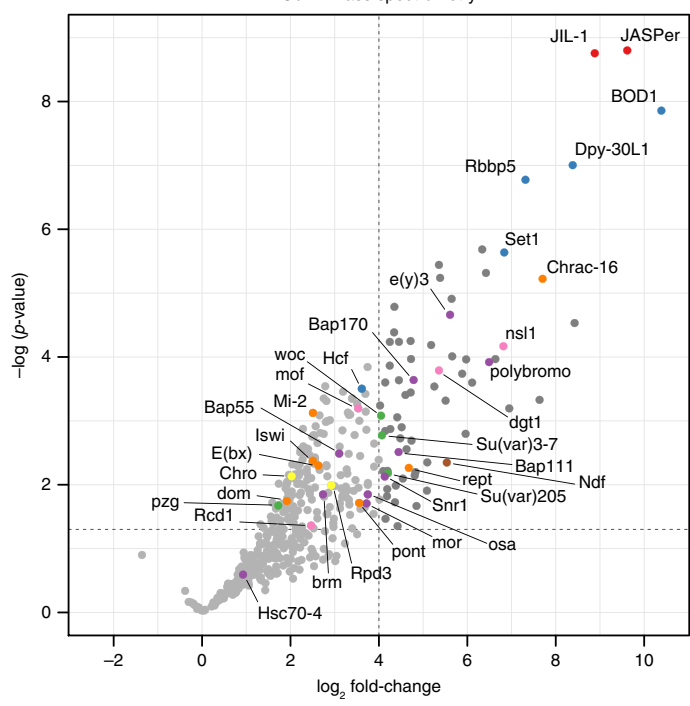

b

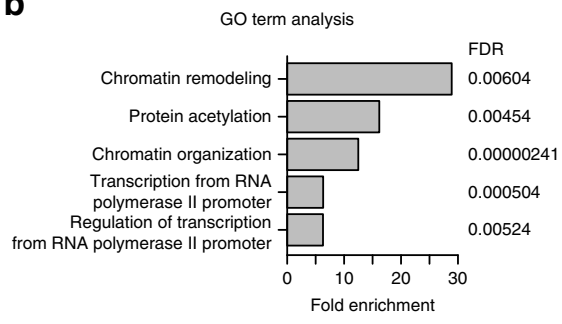

C

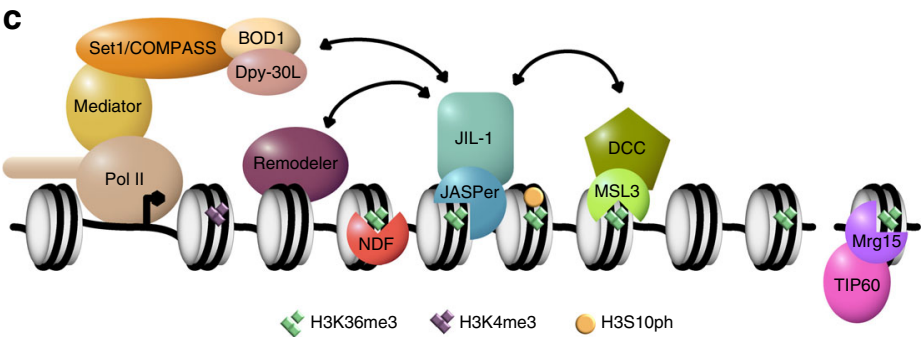

Fig. 7 The JASPer interaction network and other H3K36me3 binding proteins and complexes in Drosophila melanogaster. a Volcano plot of IP-MS showing $-\log _{10}$ ( $p$-values) against mean $\log _{2}$ fold-change in $\alpha$-JASPer IP $(n=6)$ versus control IP $(n=5)$. Significantly enriched ( $p$-value $<0.05$ and log 2 fold-change $>4)$ proteins $(n=69)$ are highlighted in dark gray. JIL-1 and JASPer are marked in red, Set1/COMPASS complex members in blue, PBAP/Brm complex members in purple, other proteins involved in chromatin remodeling in orange, NSL complex members in pink, Su(var)3-7, Su(var)205, woc and pzg in green, Chro and Rpd3 in yellow and NDF in brown. b Bar plot showing GO term enrichment of significantly enriched proteins shown in a. The five statistically significantly ( $\mathrm{fdr}<0.01$ ) most enriched GO terms are shown. c Model of JJ-complex binding at H3K36me3 marked gene bodies and interactions with other complexes. Interactions presented here are indicated by arrows. Other known H3K36me3 binding proteins (NDF and Mrg15) are drawn at the lower side. We propose that phosphorylation by JIL-1 kinase is tightly regulated in space and time in part by its partner JASPer which stabilizes and anchores the kinase to active genes and telomeric transposons by binding to H3K36me3 nucleosomes via its PWWP domain. Regulation by JASPer affects any potential phosphorylation by JIL-1, in particular H3Ser10 phosphorylation which is involved in inhibition of heterochromatinisation.

In summary, we found that the JJ-complex associated with Set1/COMPASS and several nucleosome remodeling complexes. These interactions provide links for understanding the regulation of chromatin structure and function through the JJ-complex.

\section{Discussion}

We showed that JIL-1 kinase forms as stable complex with a so far uncharacterized protein encoded by CG7946. We named the protein JASPer (JIL-1 Anchoring and Stabilizing Protein). Together the proteins form the JASPer/JIL-1 (JJ)-complex (Fig. 1), which is the major form of JIL-1 kinase in vivo, since JIL1 is unstable in the absence of JASPer (Fig. 2). The interaction is mediated by a short stretch of conserved residues within JIL-1's CTD containing a conserved FxGF motif and the LEDGF domain of JASPer. This interaction mode seems to be conserved throughout the animal kingdom, since the human JASPer ortholog PSIP1 (or LEDGF/p75) binds via its LEDGF/IBD (Integrase Binding Domain) domain to various interaction partners, including HIV integrase, MLL1-MENIN complex and IWS1 containing the conserved FxGF motif ${ }^{29}$. These interactions may also trigger deleterious targeting. For example, PSIP1 is hijacked by the HIV integrase to ensure integration of the viral genome in active chromatin, or PSIP1 mis-targets the MLL1 fusion in mixed-lineage leukemia (MLL), inducing malignant transformation. Interestingly, the stability of the interaction with MLL1 is regulated through phosphorylation ${ }^{29}$. We found similar proteins and complexes associated with the JJ-complex under stringent IPMS conditions. The most prominent interactors, Dpy-30L1, BOD1, Rbbp5, and Set1 are subunits of the Set1/COMPASS complex, which is related to the human MLL complexes. Several subunits of the PBAP/Brm complex, as well as other remodeling complexes are also enriched with the JJ-complex and contribute to the most enriched GO term (Fig. 7).

We suggest that JASPer drives the targeting of JIL-1 to active chromatin through its PWWP domain. The protein binds DNA and RNA, as well as $\mathrm{H} 3 \mathrm{~K} 36 \mathrm{me} 3$ nucleosomes in vitro. We propose that the recruitment of the JJ-complex to the body of active genes enriched in $\mathrm{H} 3 \mathrm{~K} 36 \mathrm{me} 3$ (Fig. 3) is the main recruitment mode of JIL-1 kinase to chromatin, but we do not exclude that additional binding modes are relevant at promoters and enhancers as described earlier ${ }^{8,21}$. Those binding modes could implicate interactions with other chromatin complexes, RNA or DNA. Recently, the protein PWWP2A protein was described to bind 
H2A.Z-containing nucleosomes at the 5' end of transcribed genes, as well as active gene bodies decorated with $\mathrm{H} 3 \mathrm{~K} 36 \mathrm{me} 3$ using two different binding modules ${ }^{35,53}$

The same targeting principle by JASPer binding via its PWWP domain to H3K36me3 may be used to recruit JIL-1 to telomeric HTT transposons (Fig. 6). However, it is not clear if those transposons acquire $\mathrm{H} 3 \mathrm{~K} 36 \mathrm{me} 3$ through the Set2-dependent methylation associated with elongating RNA Pol II, as coding genes $\mathrm{do}^{54}$ or by another mechanism.

The recombinant JJ-complex has a strong kinase activity towards $\mathrm{S} 10$ on isolated $\mathrm{H} 3$ in vitro but the efficiency of phosphorylating $\mathrm{H} 3 \mathrm{~S} 10$ in nucleosomes is very low (Supplementary Fig. 4). H3K36me3 is essential to bring JIL-1 to active chromatin, but is not sufficient to unleash its kinase activity upon nucleosomes in vitro. We speculate that JIL-1 may need to be activated by specific signals generated within chromatin or downstream of a signaling pathway, similarly to its orthologous kinases MSK1/2 [for review see ref. ${ }^{55}$ ]. However, the nucleosome may not be the physiological substrate for JIL-1. During the course of transcription, nucleosomes are disassembled and evicted histones associate with various chaperones to be reassembled after the passage of the polymerase [for review see ref. ${ }^{56}$ ]. Thus, H3 substrate for JIL-1 phosphorylation in vivo could also be any intermediate, occurring during the transcription process.

Methylation of $\mathrm{H} 3 \mathrm{~K} 36$ at active chromatin has pleiotropic functions in various model organisms, such as repression of spurious transcription, alternative splicing, DNA repair and recombination [for review see ref. ${ }^{57}$ ]. We summarize in Fig. 7c, the different factors known to localize to H3K36me3 chromatin in D. melanogaster. Recently, a H3K36R mutant of the replication-dependent $\mathrm{H} 3$ in $D$. melanogaster, resulting in almost complete loss of $\mathrm{H} 3 \mathrm{~K} 36 \mathrm{me} 3$, showed that this residue is essential for viability ${ }^{58}$ and triggers dysregulation of transcript levels mostly by post-transcriptional mechanisms ${ }^{59}$. Our results are compatible with an indirect function of H3K36me3 on transcriptional output.

It is attractive to speculate that JIL-1 may affect gene activity indirectly through installation of a phospho-methyl switch in interphase. Accordingly, phosphorylation of $\mathrm{H} 3 \mathrm{~S} 10$ prevents methylation of $\mathrm{H} 3 \mathrm{~K} 9$, which would hinder heterochromatinization by inhibiting further $\mathrm{H} 3 \mathrm{~K} 9$ methylation and $\mathrm{HP} 1$ binding 9,14 . We found that in JASPer ${ }^{\text {cw2 } / c w 2}$ deficient flies, as already described for $J I L-1^{z 2 / z 2}$ null flies, heterochromatin histone marks and HP1 spread from the chromocenter especially to the X chromosome on polytene nuclei of salivary glands (Fig. 2d). JIL-1 depletion in S2 cells induces a small but significant increase of H3K9me2 on genes (Supplementary Figs. 8, 9c) in S2 cells. The quantification of $\mathrm{H} 3 \mathrm{~K} 9 \mathrm{me} 2$ on transposons of the X chromosome is not yet possible due to the lack of annotation of these elements. However, transposons of the HTT arrays, which are in a heterochromatic environment, clearly acquire $\mathrm{H} 3 \mathrm{~K} 9 \mathrm{me} 2$. The apparent difference in the magnitude of $\mathrm{H} 3 \mathrm{~K} 9 \mathrm{me} 2$ spreading between the salivary gland cells and S2 cells might be due to several reasons. First, the strong mitotic H3S10ph by Aurora $\mathrm{B}$ kinase might reset the system at each cell division in cultured cells. Second, the endoreplication in salivary gland cells might exacerbate the antagonism between $\mathrm{H} 3 \mathrm{~S} 10 \mathrm{ph}$ and $\mathrm{H} 3 \mathrm{~K} 9 \mathrm{me} 2$ due to replication as described in $\mathrm{mESCs}^{7}$. Third, the absolute amount of $\mathrm{H} 3 \mathrm{~K} 9 \mathrm{me} 2$ spreading on the $\mathrm{X}$ chromosome, although evident on polytene chromosome preparations might be low, as observed in $\mathrm{mESCs}^{7}$.

The most prominent effect in our RNA-seq experiments is the specific reduction of X-chromosomal transcription in male S2 but not female Kc cells. Because the JJ-complex is also enriched on the $\mathrm{X}$ chromosome in male cells in a JIL-1- and dosage compensation dependent manner (Fig. 4), there may be a link either to the specific compensation mechanism established by the DCC or to a more general compensation mechanism known to occur in response to variation in copy number of genes. Such a 'generic' compensation mechanism has been described in many eukaryotes, as well as in various Drosophila cell lines ${ }^{60,61}$. There are two main arguments for the first scenario: the enrichment of the JJcomplex on the $\mathrm{X}$ chromosome in males depends on the DCC and decreases with increasing distance to HAS [ref. ${ }^{6}$ and Supplementary Fig. 8b] and we documented a weak but consistent interaction of the recombinant JJ-complex and partial DCC (Supplementary Fig. 8c).

Finally, we can imagine a role for the JJ-complex in the context of safeguarding genome stability threatened by R-loop formation. The presence of $\mathrm{H} 3 \mathrm{~S} 10 \mathrm{ph}$ at transcribed regions has been related to the formation of R-loops, and proposed to be important to prevent genomic instability ${ }^{62,63}$. In Drosophila cells, almost 50\% of R-loops detected by GRID-seq appear within genes ${ }^{64}$. The formation of R-loops and associated proteins could trigger the activation of JJ-complex for efficient H3S10 phosphorylation. Ectopic H3S10ph by JIL-1 correlates with large-scale chromatin opening in vivo ${ }^{65}$ although $\mathrm{H} 3 \mathrm{~S} 10$ ph per se has no effect on chromatin structure in vitro ${ }^{66}$. Conceivably, other proteins than H3 may be relevant substrates for the JJ-complex. Chromatin remodeling enzymes, which correspond to the most significantly overrepresented GO term in our unbiased IP-MS analysis of the JJ-complex interaction network would be good candidates for such regulation.

In summary, we showed that JASPer is essential for JIL-1 function: it stabilizes JIL-1 and recruits it to transcribed chromatin. Future goals will be to unravel the signaling events that lead to activation of the JJ-complex, its non-histone substrates and role in modulating chromatin structure and function.

\section{Methods}

Cell culture and RNAi. S2-DRSC (DGRC stock \# 181), Kc167 (DGRC stock \# 1) cells were cultured in Schneider's Drosophila Medium (Thermo Fisher), supplemented with $10 \%$ heat-inactivated Fetal Bovine Serum (Sigma-Aldrich), 100 units/ $\mathrm{mL}$ penicillin and $0.1 \mathrm{mg} / \mathrm{mL}$ streptomycin (Sigma-Aldrich) at $26^{\circ} \mathrm{C}$. RNAi against target genes in S2 and Kc cells for ChIP-seq was performed for 7 days in 1 or 2 flasks $\left(75 \mathrm{~cm}^{2}\right)$ seeded with 12 million cells and treated with $50 \mu \mathrm{g}$ dsRNA/flask after a wash in serum free medium ${ }^{46}$. Fresh medium was added at day 5 to sustain growth. For RNAi against target genes in S2 and Kc cells for RNA-seq, cells were washed with serum-free medium and $10 \mu \mathrm{g}$ dsRNA per $10^{6}$ cells at a concentration of $10 \mu \mathrm{g} / \mathrm{mL}$ in serum-free medium ( $10^{6}$ cells in 6 -well plate) was added, incubated for $10 \mathrm{~min}$ at room temperature (RT) with slight agitation and further $50 \mathrm{~min}$ at $26^{\circ} \mathrm{C}$. Two volumes of complete growth medium were added and cells were incubated for 3 days at $26^{\circ} \mathrm{C}$. At day 3, cells were split, reseeded and retreated as at day 1. Cells were incubated for further 4 days at $26^{\circ} \mathrm{C}$. dsRNA was generated from PCR products obtained using the following forward and reverse primers (separated by comma):

jasper RNAi \#1: TTAATACGACTCACTATAGGGAGAATGGGTAAGGAA, TTAATACGACTCACTATAGGGAGAGGAGGTGCTAGT;

jasper RNAi \#2:

TTAATACGACTCACTATAGGGAGATGGAGAACGCCCGCAAAGAA, TTAATACGACTCACTATAGGGAGATTGCCCACATACCGGCGAAG; jil-1 RNAi \#1: TTAATACGACTCACTATAGGGAGACAGCAGCGTCG, TTAATACGACTCACTATAGGGAGATTGGAACTGAT;

jil-1 RNAi \#2:

TTAATACGACTCACTATAGGGAGACAGTGGTTATCCCTTCGCA, TTAATACGACTCACTATAGGGAGATACCGCGGAGAATGAATACC; gst RNAi:

TTAATACGACTCACTATAGGGAGAATGTCCCCTATACTAGGTTA, TTAATACGACTCACTATAGGGAGAACGCATCCAGGCACATTG; $g f p$ RNAi: TTAATACGACTCACTATAGGGTGCTCAGGTAGTGGTTGTCG, TTAATACGACTCACTATAGGGCCTGAAGTTCATCTGCACCA; $n d f$ RNAi \# 1 :

TTAATACGACTCACTATAGGGAGAATCGGTCAAGTCGACAAAGG,

TTAATACGACTCACTATAGGGAGATCATTCCAAGACCCAGGAAGC; $n d f$ RNAi \#2:

TTAATACGACTCACTATAGGGAGACCGAAAGCAAAGTCCGTGG, TTAATACGACTCACTATAGGGAGAAACCTTGTGACCCGTGTAGA; D. virilis $79 \mathrm{f} 7 \mathrm{Dv} 3$ cells $^{67}$ (kind gift of B. V. Andrianov) were cultured in Schneider's Drosophila Medium (Thermo Fisher), supplemented with $5 \%$ heat- 
inactivated Fetal Bovine Serum (Sigma-Aldrich), 100 units/mL penicillin, and 0.1 $\mathrm{mg} / \mathrm{mL}$ streptomycin (Sigma-Aldrich) at $26^{\circ} \mathrm{C}$.

Sf21 cells (Thermo Fischer) were cultured in SF900 II SFM (Thermo Fisher), supplemented with $10 \%$ heat-inactivated Fetal Bovine Serum (Sigma-Aldrich), 0.1 $\mathrm{mg} / \mathrm{mL}$ gentamicin (Sigma-Aldrich) at $26^{\circ} \mathrm{C}$.

Recombinant protein expression and purification. For purification of GSTJASPer fusion protein, the coding sequence of JASPer (CG7946-RA) from EST clone LD23804 was cloned into PGEX-4T2. GST-JASPer was expressed in E. coli Rosetta 2 (DE3) (Merck) and purified using Glutathione Sepharose High Performance beads (GE Healthacare) for antibody generation. For all biochemical assays, we used the baculovirus expression system in Sf21 cells. For purification of recombinant JASPer and aromatic cage mutant (Y23A and W26A) by FLAG-tag affinity chromatography, the coding sequence of JASPer was directly fused to a C-terminal coding sequence of FLAG affinity tag and cloned into pFBDM under control of the polyhedrin promoter 22 . For dual expression of the JJ-complex, we cloned FLAG-JIL-1 or active sites mutant (D407A and D759A) and fragments thereof into PFBDM under the control of the polyhedrin promoter together with untagged JASPer or aromatic cage mutant (Y23A and W26A) and fragments thereof under the control of the p10 promoter. An N-terminal FLAG tag was directly cloned in front of the JIL-1 gene (JIL-1-RA) ${ }^{16}$. FLAG-MSL3 was expressed from pFastBacl as described ${ }^{68}$. FLAG-MSL1 was expressed from pFBDM under the control of the polyhedrin promoter together with untagged MSL2 under the control of the p10 promoter (Müller et al., manuscript in preparation).

The JJ-complex and JASPer were expressed in Sf21 cells and purified by FLAGtag affinity chromatography, as previously described ${ }^{69}$ with minor modifications. In brief, Sf2 1 cells at $10^{6}$ cells $/ \mathrm{mL}\left(250 \times 10^{6}\right.$ cells $)$ were infected 1:1000 (v/v) with baculovirus, expressing JJ-complex or JASPer-FLAG. After $72 \mathrm{~h}$, cells were harvested and washed once in Phosphate buffered saline (PBS), frozen in liquid nitrogen and stored at $-80^{\circ} \mathrm{C}$. To lyse, cells were rapidly thawed, resuspended in $5 \mathrm{~mL}$ Lysis Buffer per $50 \mathrm{~mL}$ of culture (50 mM HEPES pH 7.6, $400 \mathrm{mM} \mathrm{NaCl}$, $1 \mathrm{mM} \mathrm{MgCl}_{2}, 5 \%$ (v/v) glycerol, 0.5\% (v/v) IGEPAL CA-360, $1 \mathrm{mM} \mathrm{DTT)}$ supplemented with cOmplete EDTA-free Protease Inhibitor Cocktail (SigmaAldrich) (PI). The suspension was sonicated for $60 \mathrm{~s}$ at $20 \%$ Amplitude (BransonSonifier) with $5 \mathrm{~s}$ 'on' and $10 \mathrm{~s}$ 'pause' cycles. Cell extract was treated with $1 \mu \mathrm{L}$ Benzonase (Merck), supplemented with $0.15 \%$ (v/v) Triton-X-100, incubated with end-over-end rotation for $30 \mathrm{~min}$ at $4{ }^{\circ} \mathrm{C}$ and spun down at $4{ }^{\circ} \mathrm{C}$ for $30 \mathrm{~min}$ at $50,000 \times g$. The supernatant was used for FLAG-tag affinity purification with $1.2 \mu \mathrm{L}$ of FLAG-M2 bead bed volume (Sigma-Aldrich) per $1 \mathrm{~mL}$ of culture. Beads were first washed thrice in 20 bed volumes of Lysis Buffer, subsequently supernatant was added and incubated with end-over-end rotation for $3 \mathrm{~h}$ at $4{ }^{\circ} \mathrm{C}$. Beads were pelleted $\left(4^{\circ} \mathrm{C}, 5 \mathrm{~min}, 500 \times \mathrm{g}\right)$ and supernatant was removed. Beads were washed twice with 20 bed volumes each of Lysis Buffer, Wash Buffer (Lysis Buffer with $1 \mathrm{M}$ $\mathrm{NaCl}$ ) and finally twice with 20 bed volumes Elution Buffer (Lysis Buffer with $200 \mathrm{mM} \mathrm{NaCl}$ ). For protein elution, beads were incubated with 0.2 bed volumes of Elution Buffer containing $5 \mathrm{mg} / \mathrm{mL}$ FLAG peptide (Sigma-Aldrich) for $10 \mathrm{~min}$ at $4{ }^{\circ} \mathrm{C}$ and subsequently 0.6 bed volumes of Elution Buffer with PI were added and incubated with end-over-end rotation for $2 \mathrm{~h}$ at $4{ }^{\circ} \mathrm{C}$. The elution step was repeated and elution fractions were combined and concentrated if needed. Protein concentration was determined using BSA standards on SDS-PAGE with Coomassie brilliant blue G250 staining. Protein samples were flash-frozen in aliquots in liquid nitrogen and stored at $-80^{\circ} \mathrm{C}$. For nucleosome IP, buffer was exchanged by adding 9 volumes of Exchange Buffer 1 (50 mM HEPES pH 7.6, $500 \mathrm{mM} \mathrm{NaCl}, 1 \mathrm{mM}$ $\mathrm{MgCl}_{2}, 10 \%$ (v/v) glycerol, $0.05 \%$ (v/v) Triton-X-100, 1 mM DTT, 0.5 mM EDTA, $2.5 \mathrm{mM}$ L-Aspartate), and concentrating with $30 \mathrm{MWCO}$ Amicon Ultra-15 (Merck) to the starting volume. The proteins were again diluted in 9 volumes of Exchange Buffer 2 (Exchange Buffer 1 with $200 \mathrm{mM} \mathrm{NaCl}$ ) and concentrated with 30 MWCO Amicon Ultra-15 (Merck).

Electro mobility shift assay. EMSA with dsDNA was performed as described in ref. ${ }^{70}$, with slight modifications. In brief, binding reactions containing $70 \mathrm{nM} 40 \mathrm{bp}$ Cy5-labeled dsDNA (CCTGGA-

GAATCCCGGTGCCGAGGCCGCTCAATTGGTCGTA) in Binding buffer (50 $\mathrm{mM}$ HEPES pH 7.6, $50 \mathrm{mM} \mathrm{NaCl}, 10 \%$ (v/v) Glycerol, $2 \mathrm{mM} \mathrm{MgCl} 2,10 \%(\mathrm{w} / \mathrm{v})$ BSA) were incubated for $10 \mathrm{~min}$ at RT. EMSA with RNA was performed as described in ref. ${ }^{33}$ with $2.5 \mathrm{nM} 123 \mathrm{nt}^{32} \mathrm{P}$-labeled roX2-123 RNA in EMSA buffer (25 mM HEPES pH 7.6, $100 \mathrm{mM} \mathrm{KCl,} 3 \mathrm{mM} \mathrm{MgCl} 2,1 \mathrm{mM}$ DTT, 5\% (v/v) glycerol, $100 \mu \mathrm{g} / \mathrm{mL}$ yeast tRNA (Sigma)) for $15 \mathrm{~min}$ at $20^{\circ} \mathrm{C}$. The protein:DNA/RNA complexes were resolved by native PAGE ( $4 \%$ gel in $0.5 \times$ TBE running buffer).

Generation of JASPer null mutant fly line. The JASPer null allele $c w 2$ was isolated in a screen for imprecise excisions from the EP-element line GS3268 from the Kyoto Stock Center using standard techniques ${ }^{71}$ and as previously described ${ }^{1}$. The approximate breakpoint locations determined by PCR-analysis are shown in Fig. 2c.

Antibodies. Polyclonal antibodies against JIL-1, a-JIL-1 R69, and R70 were described in ref. ${ }^{6}$ and Hope in ref. ${ }^{20}$. GST-JASPer (1-475) was used to generate polyclonal antibodies ( $\alpha$-JASPer GP13 and GP14) in guinea pigs (Eurogentech), as well as the monoclonal (E. Kremmer) antibodies $\alpha$-JASPer 6F7 and 4D8. $\alpha$-NDF was a kind gift from J. Kadonaga ${ }^{72}$ and GST-MSL3 was used to generate polyclonal antibodies ( $\alpha$-MSL3) in guinea pig (Pineda Antikörper-Service) $)^{73}$. The following commercially available antibodies were used: $\alpha-\mathrm{H} 3 \mathrm{~K} 36 \mathrm{me} 3$ (Abcam, ab9050), $\alpha$ FLAG (Sigma, F3161), $\alpha-H 3 K 9 m e 2$ (Abcam, ab1220), $\alpha-H 3$ (Cell Signaling, 9715), a-H3S10ph (Cell Signaling, 9701), a-H4 (Abcam, ab10158), a-H4K16ac (Millipore, 07-329), $\alpha$-Tubulin (Sigma-Aldrich, T9026), and $\alpha$-LacI (Millipore, 05-503). For western blots, working concentrations of antibodies were empirically determined (polyclonal sera: 1/500-1/5000; monoclonal culture supernatants: $1 / 2-1 / 10$ ). All antibody dilutions in PBS 3\% BSA were reused several times. For detection either the infra-red based Odyssey system (Li-Cor) or the ECL based chemiluminescence system with Chemidoc Touch (Bio-rad) were used.

\section{Immunofluorescence microscopy of polytene chromosomes. Immuno-}

fluorescence microscopy analysis of polytene chromosome squash preparations was performed as described in ref. ${ }^{74}$. LacI-tagged JIL-1 constructs and the Lac operator insertion line P11.3 were described in refs. ${ }^{31,65}$. These lines include: LacI-JIL-1-FL, LacI-JIL-1-CTD, and LacI-JIL-1- $\triangle C T D$. GAL4-expression was driven by generating recombinant lines with Sgs3-GAL4 and da-GAL4 drivers obtained from the Bloomington Stock Center. Antibody labeling protocols were as in ref. ${ }^{75}$. DNA was visualized by staining with Hoechst 33258 (Molecular Probes) in PBS. The appropriate species-specific and isotype-specific Texas Red-conjugated, TRITCconjugated, and FITC-conjugated secondary antibodies (Cappel/ICN, Southern Biotech) were used (1:200 dilution) to visualize primary antibody labeling. Mounting of the preparations was in $90 \%$ glycerol including $5 \% \mathrm{n}$-propyl gallate Epifluorescence optics were used to examine the preparations on a Zeiss Axioskop microscope. Images were obtained and digitized using a Spot CCD camera. Photoshop (Adobe) was used to pseudocolor, image process, and merge images. Nonlinear adjustments were performed for some images of Hoechst labeling for the best chromosomal visualization.

JASPer identification. Nuclear extract from fly embryos were prepared from $12 \mathrm{~h}$ embryo collections as described in ref. ${ }^{76}$. For preparative immunoprecipitation (IP), $300 \mu \mathrm{g}$ nuclear embryo extract $0-12 \mathrm{~h}$ at a concentration of $3 \mathrm{mg} / \mathrm{mL}$ in HEMG100 buffer (25 mM HEPES pH 7.6, $100 \mathrm{mM} \mathrm{KCl,} \mathrm{10 \%} \mathrm{(v/v)} \mathrm{glycerol,}$ $0.1 \mathrm{mM}$ EDTA, $12.5 \mathrm{mM} \mathrm{MgCl}_{2}$ ) were used per IP. Protein A and Protein G beads mix (1:1) (GE Healthcare) were washed with HEMG100. The diluted extract was pre-cleared with $15 \mu \mathrm{L}$ ( $30 \mu \mathrm{L} 50 \%$ slurry) Protein A:Protein G beads mix by incubating with end-over-end rotation for $1 \mathrm{~h}$ at $4^{\circ} \mathrm{C}$. Beads were pelleted and supernatant was directly used for IP. For IP, the reaction was added to $15 \mu \mathrm{L}(30 \mu \mathrm{L}$ $50 \%$ slurry) Protein A:Protein G beads (GE Healthcare) pre-coupled with antibodies. For pre-coupling, beads were washed with HEMG100 buffer and incubated with end-over-end rotation for $1 \mathrm{~h}$ at $4{ }^{\circ} \mathrm{C}$ with $2 \mu \mathrm{g}$ antibodies in HEMG100, using affinity-purified $\alpha$-JIL-1 R69 and R70 and non-specific rabbit IgG as control. Beads were washed with HEMG100, the extract was added and incubated with end-overend rotation for $1 \mathrm{~h}$ at $4^{\circ} \mathrm{C}$. Beads were spun down and washed three times with HEMG100. Proteins were eluted by incubating beads with

HEMG100 supplemented with $0.5 \%(\mathrm{~m} / \mathrm{v}) \mathrm{N}$-lauroylsacrosine with end-over-end rotation for $1 \mathrm{~h}$ at $4{ }^{\circ} \mathrm{C}$. Proteins were separated by $4-20 \%$ gradient SDS-PAGE, stained by Coomassie brilliant blue G250 staining and the most prominent band was cut out for mass spectrometry analysis.

Immunoprecipitation from embryo extracts. Nuclear extract from fly embryos were prepared from $12 \mathrm{~h}$ embryo collections as described in ref. ${ }^{76}$. For each IP, $400 \mu \mathrm{g}$ of extract was diluted to $1 \mathrm{mg} / \mathrm{mL}$ in BBN buffer $(10 \mathrm{mM}$ Tris/Cl pH 8.0 , $140 \mathrm{mM} \mathrm{NaCl}, 1 \mathrm{mM}$ EDTA, $1 \%(\mathrm{v} / \mathrm{v})$ Triton X-100, 0.1\% (v/v) Na deoxycholate, $0.1 \%(\mathrm{v} / \mathrm{v})$ IGEPAL-CA-360, $0.5 \mathrm{mM}$ DTT) supplemented with cOmplete EDTAfree Protease Inhibitor Cocktail (Sigma-Aldrich). Protein G beads (GE Healthcare) were washed thrice with 10 bed volumes BBN buffer. The diluted extract was precleared with $10 \mu \mathrm{L}(20 \mu \mathrm{L} 50 \%$ slurry) Protein G beads by incubating with endover-end rotation for $1 \mathrm{~h}$ at $4{ }^{\circ} \mathrm{C}$. Beads were pelleted at $4{ }^{\circ} \mathrm{C}$ for $5 \mathrm{~min}$ at $500 \times \mathrm{g}$ and supernatant was directly used for IP. For IP/MS analysis, IP's were performed from two independent nuclear embryo extracts with two different $\alpha$-JASPer polyclonal Sera (GP13 and GP14) and two different culture supernatants containing monoclonal antibodies (6F7 and 4D8), as negative control a non-specific serum or culture medium of hybridomas was used. The supernatant was added to $25 \mu \mathrm{L}$ (50 $\mu \mathrm{L} 50 \%$ slurry) Protein G beads (GE Healthcare) pre-coupled with antibodies. For pre-coupling, beads were washed thrice with BBN buffer and incubated with end-over-end rotation for $3-4 \mathrm{~h}$ at $4{ }^{\circ} \mathrm{C}$ with $1.5 \mathrm{~mL}$ culture supernatant containing monoclonal antibody and culture medium of hybridomas as control or $2 \mu \mathrm{L}$ serum in BBN buffer. Beads were washed thrice with BBN buffer, the extract was added and incubated with end-over-end rotation for $3-4 \mathrm{~h}$ at $4{ }^{\circ} \mathrm{C}$. Beads were spun down and washed thrice with 40 bed volumes BBN buffer and twice in 10 bed volumes $50 \mathrm{mM}$ Tris/Cl pH 7.5 by incubating with end-over-end rotation for $10 \mathrm{~min}$ at $4^{\circ} \mathrm{C}$ before handing over to the proteomics core facility.

Mass spectrometry and data analysis. Whole IPs were used for trypsin digestion and mass spectrometry (IP/MS) identification of binding partners. For LC-MS/MS purposes, desalted peptides were injected in an Ultimate 3000 RSLCnano system 
(Thermo), separated in a 15-cm analytical column ( $75 \mu \mathrm{m}$ ID home-packed with ReproSil-Pur C18-AQ $2.4 \mu \mathrm{m}$ from Dr. Maisch) with a 50-min gradient from 5 to $60 \%$ acetonitrile in $0.1 \%$ formic acid. The effluent from the HPLC was directly electrosprayed into a Q Exactive HF (Thermo) operated in data-dependent mode to automatically switch between full scan MS and MS/MS acquisition. Survey full scan MS spectra (from $\mathrm{m} / \mathrm{z} 375-1600$ ) were acquired with resolution $R=60,000$ at $\mathrm{m} / z 400$ (AGC target of $3 \times 10^{6}$ ) and MS/MS spectra with resolution 15,000 at $\mathrm{m} / \mathrm{z}$ 400 (AGC target of $1 \times 10^{5}$ ). The 10 most intense peptide ions with charge states between 2 and 5 were sequentially isolated to a target value of $1 \times 10^{5}$, and fragmented at $27 \%$ normalized collision energy. Typical MS conditions were: spray voltage, $1.5 \mathrm{kV}$; no sheath and auxiliary gas flow; heated capillary temperature, $250^{\circ} \mathrm{C}$; ion selection threshold, 33.000 counts. MaxQuant version 1.5.2.877 was used to identify proteins and to quantify by $\mathrm{iBAQ}$ with the following parameters: Database, UP000000803_7227_Drome_20160809; MS tol, 10 ppm; MS/MS tol, 20 ppm; Peptide FDR, 0.1; Protein FDR, 0.01 Min. peptide Length, 5; Variable modifications, Oxidation (M); Fixed modifications, Carbamidomethyl (C); Peptides for protein quantitation, razor and unique; Min. peptides, 1; Min. ratio count, 2. The resulting "proteinGroups.txt" file was used for further downstream analysis using DEP version 1.4.0 $0^{72}(\mathrm{R})$ and MSnbase version 2.8.1 $1^{78}(\mathrm{R})$. First, reverse proteins and potential contaminants were removed. The data was filtered for missing values allowing maximally one missing value in at least one condition by calling the function filter_missval (R) (parameter thr $=1$ ). Missing values in control IP samples were considered as missing not at random and imputed using the quantile regression imputation of left-censored data (QRILC) method by calling the function impute (R) (parameter method = "QRILC"). Missing values in the IP samples were considered as missing at random and imputed using the quantile k-nearest neighbor $(\mathrm{knn})$ method by calling the function impute $(\mathrm{R})$ (parameter method = "knn"). To test for statistically significant differentially enriched proteins, the function test_diff (R) was called including condition and sample variables. Proteins were considered as statistically significant enriched with $p$-value $<0.05$ and $\log _{2}$ fold enrichment $>4$. GO term analysis of statistical significant enriched proteins was performed with http://www.pantherdb.org using the PANTHER Overrepresentation Test analysis type and PANTHER GO-Slim Biological Process GO terms ${ }^{79,80}$. Protein-Protein interaction network on known interactions of statistically significantly enriched proteins was generated using Cytoscape version 3.7.0.01 and STRING database ${ }^{82}$.

Mapping of protein interactions by co-immunoprecipitation. Protein-protein interactions were studied with recombinant proteins, in extracts from baculovirus infected Sf21 cells (see also the section of recombinant protein expression). Interaction domains in JASPer and JIL-1 were mapped by co-IP of various truncation mutants using the N-terminal FLAG-tag in JIL-1. JASPer derivatives were all untagged. Interaction of JJ-complex with the core MSL complex (MSL1, MSL2, and MSL3) was analyzed by co-IP from Sf21 cell extracts as described in ref. ${ }^{46}$. In brief, the expression level of all FLAG tagged proteins was assessed by $\alpha$-FLAG western blot of single extracts and extracts were mixed in order to achieve similar final concentration of the recombinant proteins. IP's were performed with antibodies specific for the specified bait.

\section{Mononucleosomes, 12-mer nucleosome arrays, and kinase assays. Mono-} nucleosomes and nucleosome arrays used as substrates for the kinase assays were prepared by salt gradient dialysis as described ${ }^{34,83}$. Briefly, histone octamers (wt and H3K36me3), (biotinylated) scavenger MMTV DNA, and the corresponding $601 \mathrm{DNA}^{34,84}$ in $20 \mathrm{mM}$ Tris/HCl, $2 \mathrm{M} \mathrm{KCl}, 0.1 \mathrm{mM}$ EDTA pH 7.5 at $4{ }^{\circ} \mathrm{C}$ were dialyzed into $200 \mathrm{~mL}$ nucleosome start buffer $(10 \mathrm{mM}$ Tris/ $\mathrm{HCl}, 1.4 \mathrm{M} \mathrm{KCl}, 1 \mathrm{mM}$ DTT, $0.1 \mathrm{mM}$ EDTA pH 7.5 at $\left.4{ }^{\circ} \mathrm{C}\right)$ for $1 \mathrm{~h} .330 \mathrm{~mL}$ nucleosome end buffer $(10$ $\mathrm{mM}$ Tris/ $\mathrm{HCl}, 10 \mathrm{mM} \mathrm{KCl}, 1 \mathrm{mM}$ DTT, $0.1 \mathrm{mM}$ EDTA pH 7.5 at $4{ }^{\circ} \mathrm{C}$ ) was added overnight at $4{ }^{\circ} \mathrm{C}$ using a peristaltic pump (rate $1 \mathrm{~mL} / \mathrm{min}$ ). Subsequently, two additional dialysis steps $(4 \mathrm{~h}$ and $2 \mathrm{~h}$ ) were performed using $200 \mathrm{~mL}$ nucleosome end buffer. The samples were centrifuged $\left(17,000 \times g, 4^{\circ} \mathrm{C}, 10 \mathrm{~min}\right)$ and the supernatant isolated. Mononucleosome samples were treated with streptavidincoated magnetic beads (New England Biolabs) to deplete the biotinylated MMTV DNA and MMTV nucleosomes. All nucleosome arrays were purified by selective $\mathrm{MgCl}_{2}$ precipitation ${ }^{85}$

Recombinant $\mathrm{H} 3$ was prepared from inclusion bodies as described in ${ }^{86}$. Prior to the label-free kinase assays, the ratio of JJ-complex to $\mathrm{H} 3$ was determined by radioactive kinase assays using $\gamma$-ATP and $50 \mu \mathrm{M}$ non-radioactive ATP in $20 \mu \mathrm{L}$ total reaction volume as described earlier ${ }^{6}$. Using 2.5 pmol of JJ-complex and 10 pmol of $\mathrm{H} 3$ per assay yielded an incorporation of 1 phosphate per $\mathrm{H} 3$ molecule. We used the same conditions in the label-free kinase assays with $1 \mathrm{mM}$ of nonradioactive ATP. All reactions were performed in parallel with JJ-complex containing wild type kinase and JJ-complex containing the kinase dead mutant, which is inactive because mutated at both active sites (D407A and D759A). For quantification purposes, we loaded $0.8,1.6$, and $3.2 \%$ of the reaction performed with isolated $\mathrm{H} 3$, corresponding to $1.2,2.4$, and $4.8 \mathrm{ng}$, respectively and $30 \%(3.3$ $\mathrm{pmol}$ ) of the reactions performed with the different types of nucleosomes. The quantitative $\mathrm{H} 3 \mathrm{~S} 10$ ph detection and the loading controls ( $\mathrm{H} 4$ and $\mathrm{H} 3 \mathrm{~K} 36 \mathrm{me} 3$ ) were achieved using IR-coupled secondary antibodies and Odyssey Imaging System (LI-COR)
Nucleosome pull-down. Nucleosome library preparation, pull-down experiments and data analysis were performed as described in ref. ${ }^{34}$. Per pull-down reaction, 1.5 pmol of JASPer was used for JASPer-FLAG wt and aromatic cage mutant and for wt and aromatic cage mutant of JJ-complex and pre-coupled to $5 \mu \mathrm{L}$ FLAG-M2 beads (Sigma-Aldrich) $(10 \mu \mathrm{L} 50 \%$ slurry $)$ in Binding buffer $(20 \mathrm{mM}$ Tris/Cl $\mathrm{pH}$ 7.5, $50 \mathrm{mM} \mathrm{NaCl}, 5 \mathrm{mM}$ EDTA, $0.1 \%$ (v/v) TWEEN 20). The protein pre-coupled to beads was incubated with 1.38 pmol nucleosome library containing 115 nucleosome types ( $12 \mathrm{fmol}$ per nucleosome type) in a total of $200 \mu \mathrm{L}$ Binding buffer for $4 \mathrm{~h}$ at $4{ }^{\circ} \mathrm{C}$ with end-over-end rotation. Beads were washed four times with 40 bed volumes $(200 \mu \mathrm{L})$ Binding buffer and DNA eluted by Proteinase K digestion and purified using a QIAGEN PCR purification kit for further library preparation and sequencing.

ChIP-seq. ChIP-seq on MNase-digested chromatin and sonicated chromatin was performed as previously described ${ }^{46,87}$. For spike-in ChIP-seq on MNase-digested chromatin in combination with mild sonication, $\mathrm{S} 2$ cells $\left(\sim 3 \times 10^{8}\right.$ cells $)$ after RNA were harvested and cross-linked with $1 \%$ formaldehyde for $8 \mathrm{~min}$ by adding $1 \mathrm{~mL}$ $10 \times$ fixing solution (50 mM HEPES $\mathrm{pH} 8.0,100 \mathrm{mM} \mathrm{NaCl}, 1 \mathrm{mM}$ EDTA, $0.5 \mathrm{mM}$ EGTA) with $10 \%$ formaldehyde [ $16 \%$ formaldehyde solution (w/v) methanol-fee (Thermo Fischer)] per $10 \mathrm{~mL}$ culture at RT. The reaction was stopped by adding $125 \mathrm{mM}$ glycine and incubating for $10 \mathrm{~min}$ on ice. Cells were washed twice in PBS and snap-frozen in liquid $\mathrm{N}_{2}$. For nuclei isolation, cells were rapidly thawed and resuspended in PBS supplemented with 0.5\% (v/v) Triton X-100 and cOmplete EDTA-free Protease Inhibitor Cocktail (Sigma-Aldrich) (PI) and 5\% 79f7Dv3 cells, processed as described for S2 cells without RNAi treatment, relative to S2 cells were added, volume was adjusted to $7 \times 10^{7}$ cells/mL and cells incubated for $15 \mathrm{~min}$ at 4 ${ }^{\circ} \mathrm{C}$ with end-over-end rotation. Nuclei were collected by centrifuging at $4{ }^{\circ} \mathrm{C}$ for 10 $\min$ at $2000 \times g$ and washed once in PBS. For chromatin fragmentation, nuclei were spun down at $4{ }^{\circ} \mathrm{C}$ for $10 \mathrm{~min}$ at $2000 \times \mathrm{g}$, resuspended in RIPA $(10 \mathrm{mM}$ Tris $/ \mathrm{HCl}$ pH 8.0, $140 \mathrm{mM} \mathrm{NaCl}, 1 \mathrm{mM}$ EDTA, $1 \%$ (v/v) Triton-X 100, 0.1\%(v/v) SDS, $0.1 \%$ (v/v) DOC) supplemented with PI and $2 \mathrm{mM} \mathrm{CaCl}_{2}$ at $7 \times 10^{7}$ cells $/ \mathrm{mL}$ and digested in $1 \mathrm{~mL}$ aliquots by adding $0.6 \mathrm{U}$ MNase (Sigma Aldrich), resuspended in EX50 at $0.6 \mathrm{U} / \mu \mathrm{L}^{88}$, and incubated at $37^{\circ} \mathrm{C}$ for $35 \mathrm{~min}$ with slight agitation. The reaction was stopped by adding $10 \mathrm{mM}$ EGTA and placing on ice. Digested chromatin was sheared with Covaris AFA S220 using $12 \times 12$ tubes at $50 \mathrm{~W}$ peak incident power, $20 \%$ duty factor and 200 cycles per burst for $8 \mathrm{~min}$ at $5{ }^{\circ} \mathrm{C}$. Subsequent steps were performed as described in ref. ${ }^{46}$. Libraries were prepared with NEBNext Ultra II DNA Library Prep Kit for Illumina (NEB, E7645) and analyzed with 2100 Bioanalyzer with DNA 1000 kit (Agilent). Libraries were sequenced on HiSeq 1500 (Illumina) instrument yielding typically $20-25$ million 50 bp single-end reads per sample at the genomics facility.

RNA-seq. For RNA-seq, 2 million S2 cells or Kc cells after RNAi treatment were resuspended in Trizol and RNA was purified using the RNeasy Mini Kit (QIAGEN). Afterwards, $1 \mu \mathrm{g}$ of purified total RNA's was used for rRNA depletion using Ribo-Zero Gold rRNA Removal Kit (Illumnia, MRZG 12324) or NEBNext rRNA Depletion Kit (NEB, E6310). Library preparation was done according to the manufacturer's instructions with NEBNext Ultra II Directional RNA Library Prep Kit for Illumina (NEB, E7760) and analyzed with 2100 Bioanalyzer with DNA 1000 kit (Agilent). Libraries were sequenced on HiSeq 1500 (Illumina) instrument yielding typically $15-50$ million 50 bp paired-end reads per sample at the genomics facility.

NGS data analysis. Sequencing data were processed using SAMtools version 1.3.1 ${ }^{89}$, BEDtools version 2.26.090, R version 3.5.1 (http://www.r-project.org) and Bioconductor version 3.8 (http://www.bioconductor.org) using default parameters for function calls, unless stated otherwise.

Read processing. Sequence reads were aligned to the D. melanogaster release 6 reference genome (BDGP6), D. virilis FlyBase release r1.07_FB2018_05 reference genome or to $D$. melanogaster transposon sequence set version 9.4.1 (BDGP), including only $D$. melanogaster transposons $(n=126)$, using Bowtie version 1.1.2 $2^{91}$ (parameter $-\mathrm{m} 1$ for D. melanogaster genome and transposon) for ChIP-seq and STAR version 2.6.0 $0^{92}$ (parameters --quantMode TranscriptomeSAM GeneCounts, --outFilterMultimapNmax 1) for RNA-seq samples. Gene and transposon quantification of RNA-seq data was performed using RSEM version 1.3.093 (parameters -bam, --paired-end, --forward-prob 0).

RNA-seq analysis. For RNA-seq analysis, genes and transposons were considered as robustly detected with raw read counts $>0$ in all samples. Further analysis was performed using DESeq2 version 1.22.1 ${ }^{94}(\mathrm{R})$, including blocking variables for batch effect. For transposon analysis, the size factors from the gene analysis were used. Genes and transposons were considered as statistical significant different between conditions with false discovery rate $(\mathrm{FDR})<0.05$ by calling the function results (R). For principle component analysis (PCA), a regularized log transformation was applied to per gene counts calling the function rlog (blind = FLASE) (R). To correct for batch effects, the function ComBat (R) was called using a design matrix modeling the RNAi variable by calling the function model.matrix (R). The function plotPCA (R) was called to perform PCA. 
Genome coverage. ChIP-seq reads were extended to $150 \mathrm{bp}$ and per base normalized genome coverage vectors were calculated as described in ref. ${ }^{95}$. For normalization using $D$. virilis spike-ins, per base coverage vectors were normalized to the sum of $D$. virilis genome coverage vectors multiplied by a factor to adjust for difference in cell number, which was calculated as the total number of reads divided by the total number $D$. virilis reads. To generate non-input normalized per base genome coverage vectors, raw coverage vectors were normalized to million mapped reads $(\mathrm{rpm})$.

Browser profiles. Browser profiles were generated by calling the function plotProfiles from tsTools version 0.1.1 (R) (https://rdrr.io/github/musikutiv/tsTools/) by using mean per base genome coverage vectors after smoothing by computing running medians on $501 \mathrm{bp}$ windows calling the function runmed (endrule = "keep") (R)

ChIP-seq analysis. Two H3K36me3 MNase ChIP-seq replicates in Kc and S2 cells each were previously published (GSE94115) ${ }^{87}$ and the two Inputs for sonication ChIP-seq (GSE119708) ${ }^{46}$. For gene-centric ChIP-seq analysis, genes were considered as inactive with mean tpm $\leq 1$ and active with mean $t p m>1$ in control cells. Heat maps of mean normalized coverages at active genes \pm 500 bp were generated calling the plotRasterHeatmap and convertToColors with using a range of 0.05 to 0.95 from tsTools version 0.1.1 (R) (https://rdrr.io/github/musikutiv/ tsTools/). Only genes $>3000$ bp were considered and the gene body (from TSS $+1000 \mathrm{bp}$ to TTS $-1000 \mathrm{bp}$ ) was scaled to $2000 \mathrm{bp}$. Exons were converted to per pase gene coverage vectors using a score of 1 . Genes were hierarchical clustered on the exon coverages by calculating the Euclidean distance by calling the function dist (R) and clustered using the 'complete' method by calling the function hclust (R). To generate density plots, kernel density estimates were calculated by calling the function density (R). For transposon analysis, the P-element and Penelope transposon were removed as they had zero counts in the input samples, leaving 124 transposons. For calculating ChIP-seq signal at transposons, aligned reads were extended to $150 \mathrm{bp}$ fragments, reads were summed up and normalized with the size factor from reads aligned to the reference genome or for spike-in ChIP-seq to $D$. virilis reference genome. ChIP-seq signal enrichment at transposons was calculated as $\log _{2}$ ratio of IP over input. Heat maps were generated by calling the function pheatmap (R) and smoothed color representation of scatter plots by calling the function smoothScatter (R).

Reporting summary. Further information on research design is available in the Nature Research Reporting Summary linked to this article.

\section{Data availability}

The sequencing data discussed in this publication have been deposited in NCBI's Gene Expression Omnibus ${ }^{96}$ and are accessible through GEO Series accession number GSE128457 and the mass spectrometry data have been deposited to the ProteomeXchange Consortium ${ }^{97}$ with the dataset identifier PXD012790. All other relevant data supporting the key findings of this study are available within the article and its Supplementary Information files or from the corresponding authors upon reasonable request. The source data of cropped images in Figs. 1a, 1b, 1d, 1e, 2c, 2e, and Supplementary Figs. 4e, 4f, 8d, 13a are provided as Source Data file. A reporting summary for this Article is available as a Supplementary Information file.

Received: 30 April 2019; Accepted: 22 October 2019;

Published online: 25 November 2019

\section{References}

1. Wang, Y., Zhang, W., Jin, Y., Johansen, J. \& Johansen, K. M. The JIL-1 tandem kinase mediates histone $\mathrm{H} 3$ phosphorylation and is required for maintenance of chromatin structure in Drosophila. Cell 105, 433-443 (2001).

2. Adams, R. R., Maiato, H., Earnshaw, W. C. \& Carmena, M. Essential roles of Drosophila inner centromere protein (INCENP) and aurora B in histone H3 phosphorylation, metaphase chromosome alignment, kinetochore disjunction, and chromosome segregation. J. Cell Biol. 153, 865-880 (2001).

3. Giet, R. \& Glover, D. M. Drosophila aurora B kinase is required for histone H3 phosphorylation and condensin recruitment during chromosome condensation and to organize the central spindle during cytokinesis. J. Cell Biol. 152, 669-682 (2001).

4. Mahadevan, L. C., Willis, A. C. \& Barratt, M. J. Rapid histone H3 phosphorylation in response to growth factors, phorbol esters, okadaic acid, and protein synthesis inhibitors. Cell 65, 775-783 (1991).

5. Cheung, P. et al. Synergistic coupling of histone $\mathrm{H} 3$ phosphorylation and acetylation in response to epidermal growth factor stimulation. Mol. Cell 5, 905-915 (2000).
6. Regnard, C. et al. Global analysis of the relationship between JIL-1 kinase and transcription. PLoS Genet. 7, e1001327 (2011).

7. Chen, C. C. L. et al. H3S10ph broadly marks early-replicating domains in interphase ESCs and shows reciprocal antagonism with $\mathrm{H} 3 \mathrm{~K} 9 \mathrm{me} 2$. Genome Res. 28, 37-51 (2018).

8. Cai, W. et al. Genome-wide analysis of regulation of gene expression and H3K9me2 distribution by JIL-1 kinase mediated histone H3S10 phosphorylation in Drosophila. Nucleic Acids Res. 42, 5456-5467 (2014).

9. Fischle, W. et al. Regulation of HP1-chromatin binding by histone $\mathrm{H} 3$ methylation and phosphorylation. Nature 438, 1116-1122 (2005).

10. Chin, H. G. et al. Sequence specificity and role of proximal amino acids of the histone $\mathrm{H} 3$ tail on catalysis of murine G9A lysine 9 histone H3 methyltransferase. Biochemistry 44, 12998-13006 (2005).

11. Rea, S. et al. Regulation of chromatin structure by site-specific histone $\mathrm{H} 3$ methyltransferases. Nature 406, 593-599 (2000).

12. Lerach, S. et al. Loss-of-function alleles of the JIL-1 kinase are strong suppressors of position effect variegation of the wm4 allele in Drosophila. Genetics 173, 2403-2406 (2006).

13. Ebert, A. et al. $\mathrm{Su}(\mathrm{var})$ genes regulate the balance between euchromatin and heterochromatin in Drosophila. Genes Dev. 18, 2973-2983 (2004).

14. Zhang, W. et al. The JIL-1 histone H3S10 kinase regulates dimethyl H3K9 modifications and heterochromatic spreading in Drosophila. Development 133, 229-235 (2006).

15. Deng, H. et al. JIL-1 and Su(var)3-7 interact genetically and counteract each other's effect on position-effect variegation in Drosophila. Genetics 185, 1183-1192 (2010).

16. Boeke, J. et al. Phosphorylation of SU(VAR)3-9 by the chromosomal kinase JIL-1. PLOS ONE 5, e10042 (2010).

17. Andreyeva, E. N., Belyaeva, E. S., Semeshin, V. F., Pokholkova, G. V. \& Zhimulev, I. F. Three distinct chromatin domains in telomere ends of polytene chromosomes in Drosophila melanogaster Tel mutants. J. Cell Sci. 118, 5465-5477 (2005).

18. Silva-Sousa, R. \& Casacuberta, E. The JIL-1 kinase affects telomere expression in the different telomere domains of Drosophila. PLoS ONE 8, e81543 (2013).

19. Silva-Sousa, R., Lopez-Panades, E., Pineyro, D. \& Casacuberta, E. The chromosomal proteins JIL-1 and Z4/Putzig regulate the telomeric chromatin in Drosophila melanogaster. PLoS Genet. 8, el003153 (2012).

20. Jin, Y. et al. JIL-1: a novel chromosomal tandem kinase implicated in transcriptional regulation in Drosophila. Mol. Cell 4, 129-135 (1999).

21. Kellner, W. A., Ramos, E., Van Bortle, K., Takenaka, N. \& Corces, V. G. Genome-wide phosphoacetylation of histone $\mathrm{H} 3$ at Drosophila enhancers and promoters. Genome Res. 22, 1081-1088 (2012).

22. Fitzgerald, D. J. et al. Protein complex expression by using multigene baculoviral vectors. Nat. Methods 3, 1021-1032 (2006).

23. Ciuffi, A. et al. A role for LEDGF/p75 in targeting HIV DNA integration. Nat. Med. 11, 1287-1289 (2005).

24. El Ashkar, S. et al. LEDGF/p75 is dispensable for hematopoiesis but essential for MLL-rearranged leukemogenesis. Blood 131, 95-107 (2018).

25. Leroux, A. E., Schulze, J. O. \& Biondi, R. M. AGC kinases, mechanisms of regulation and innovative drug development. Semin. Cancer Biol. 48, 1-17 (2018).

26. Lancaster, A. K., Nutter-Upham, A., Lindquist, S. \& King, O. D. PLAAC: a web and command-line application to identify proteins with prion-like amino acid composition. Bioinformatics 30, 2501-2502 (2014).

27. Rogers, S., Wells, R. \& Rechsteiner, M. Amino acid sequences common to rapidly degraded proteins: the PEST hypothesis. Science 234, 364-368 (1986).

28. Correa Marrero, M., van Dijk, A. D. J. \& de Ridder, D. Sequence-based analysis of protein degradation rates. Proteins 85, 1593-1601 (2017).

29. Sharma, S. et al. Affinity switching of the LEDGF/p75 IBD interactome is governed by kinase-dependent phosphorylation. Proc. Natl Acad. Sci. USA 115, E7053-E7062 (2018).

30. Deng, H. et al. The JIL-1 kinase regulates the structure of Drosophila polytene chromosomes. Chromosoma 114, 173-182 (2005).

31. Li, Y. et al. Domain requirements of the JIL-1 tandem kinase for histone H3 serine 10 phosphorylation and chromatin remodeling in vivo. J. Biol. Chem. 288, 19441-19449 (2013).

32. Rona, G. B., Eleutherio, E. C. A. \& Pinheiro, A. S. PWWP domains and their modes of sensing DNA and histone methylated lysines. Biophys. Rev. 8, 63-74 (2016).

33. Maenner, S., Muller, M., Frohlich, J., Langer, D. \& Becker, P. B. ATPdependent roX RNA remodeling by the helicase maleless enables specific association of MSL proteins. Mol. Cell 51, 174-184 (2013).

34. Dann, G. P. et al. ISWI chromatin remodellers sense nucleosome modifications to determine substrate preference. Nature 548, 607-611 (2017).

35. Link, S. et al. PWWP2A binds distinct chromatin moieties and interacts with an MTA1-specific core NuRD complex. Nat. Commun. 9, 4300 (2018).

36. Davey, C. A., Sargent, D. F., Luger, K., Maeder, A. W. \& Richmond, T. J. Solvent mediated interactions in the structure of the nucleosome core particle at 1.9 a resolution. J. Mol. Biol. 319, 1097-1113 (2002). 
37. Stutzer, A. et al. Modulations of DNA contacts by linker histones and posttranslational modifications determine the mobility and modifiability of nucleosomal H3 tails. Mol. Cell 61, 247-259 (2016).

38. Eidahl, J. O. et al. Structural basis for high-affinity binding of LEDGF PWWP to mononucleosomes. Nucleic Acids Res. 41, 3924-3936 (2013).

39. van Nuland, R. et al. Nucleosomal DNA binding drives the recognition of H3K36-methylated nucleosomes by the PSIP1-PWWP domain. Epigenet. Chromatin 6, 12 (2013).

40. Straub, T., Zabel, A., Gilfillan, G. D., Feller, C. \& Becker, P. B. Different chromatin interfaces of the Drosophila dosage compensation complex revealed by high-shear ChIP-seq. Genome Res. 23, 473-485 (2013).

41. Kharchenko, P. V. et al. Comprehensive analysis of the chromatin landscape in Drosophila melanogaster. Nature 471, 480-485 (2011).

42. Bell, O. et al. Accessibility of the Drosophila genome discriminates PcG repression, H4K16 acetylation and replication timing. Nat. Struct. Mol. Biol. 17, 894-900 (2010).

43. Shogren-Knaak, M. et al. Histone H4-K16 acetylation controls chromatin structure and protein interactions. Science 311, 844-847 (2006).

44. Orlando, D. A. et al. Quantitative ChIP-Seq normalization reveals global modulation of the epigenome. Cell Rep. 9, 1163-1170 (2014).

45. Jin, Y., Wang, Y., Johansen, J. \& Johansen, K. M. JIL-1, a chromosomal kinase implicated in regulation of chromatin structure, associates with the male specific lethal (MSL) dosage compensation complex. J. Cell Biol. 149, 1005-1010 (2000).

46. Albig, C. et al. Factor cooperation for chromosome discrimination in Drosophila. Nucleic Acids Res. 47, 1706-1724 (2019).

47. Mohan, M. et al. The COMPASS family of H3K4 methylases in Drosophila. Mol. Cell Biol. 31, 4310-4318 (2011).

48. Wang, L. et al. A cytoplasmic COMPASS is necessary for cell survival and triple-negative breast cancer pathogenesis by regulating metabolism. Genes Dev. 31, 2056-2066 (2017).

49. van Nuland, R. et al. Quantitative dissection and stoichiometry determination of the human SET1/MLL histone methyltransferase complexes. Mol. Cell Biol. 33, 2067-2077 (2013).

50. Rath, U. et al. The chromodomain protein, Chromator, interacts with JIL-1 kinase and regulates the structure of Drosophila polytene chromosomes. J. Cell Sci. 119, 2332-2341 (2006).

51. Wang, C. I. et al. Chromatin proteins captured by ChIP-mass spectrometry are linked to dosage compensation in Drosophila. Nat. Struct. Mol. Biol. 20 , 202-209 (2013).

52. Fei, J. et al. NDF, a nucleosome-destabilizing factor that facilitates transcription through nucleosomes. Genes Dev. 32, 682-694 (2018).

53. Zhang, T. et al. A variant NuRD complex containing PWWP2A/B excludes $\mathrm{MBD} 2 / 3$ to regulate transcription at active genes. Nat. Commun. 9, 3798 (2018).

54. Carrozza, M. J. et al. Histone H3 methylation by Set2 directs deacetylation of coding regions by Rpd3S to suppress spurious intragenic transcription. Cell 123, 581-592 (2005).

55. Reyskens, K. M. \& Arthur, J. S. Emerging roles of the mitogen and stress activated kinases MSK1 and MSK2. Front. Cell Dev. Biol. 4, 56 (2016).

56. Hammond, C. M., Stromme, C. B., Huang, H., Patel, D. J. \& Groth, A. Histone chaperone networks shaping chromatin function. Nat. Rev. Mol. Cell Biol. 18, 141-158 (2017).

57. Wagner, E. J. \& Carpenter, P. B. Understanding the language of Lys 36 methylation at histone H3. Nat. Rev. Mol. Cell Biol. 13, 115-126 (2012).

58. McKay, D. J. et al. Interrogating the function of metazoan histones using engineered gene clusters. Dev. Cell 32, 373-386 (2015).

59. Meers, M. P. et al. Histone gene replacement reveals a post-transcriptional role for H3K36 in maintaining metazoan transcriptome fidelity. Elife 6, https://doi. org/10.7554/eLife.23249 (2017).

60. Zhang, Y. et al. Expression in aneuploid Drosophila S2 cells. PLoS Biol. 8, e1000320 (2010).

61. Lee, H. et al. DNA copy number evolution in Drosophila cell lines. Genome Biol. 15, R70 (2014).

62. Castellano-Pozo, M. et al. R loops are linked to histone H3 S10 phosphorylation and chromatin condensation. Mol. Cell 52, 583-590 (2013).

63. Garcia-Pichardo, D. et al. Histone mutants separate R loop formation from genome instability induction. Mol. Cell 66, 597-609.e595 (2017).

64. Bayona-Feliu, A., Casas-Lamesa, A., Reina, O., Bernues, J. \& Azorin, F. Linker histone $\mathrm{H} 1$ prevents R-loop accumulation and genome instability in heterochromatin. Nat. Commun. 8, 283 (2017).

65. Deng, H. et al. Ectopic histone H3S10 phosphorylation causes chromatin structure remodeling in Drosophila. Development 135, 699-705 (2008).

66. Fry, C. J., Shogren-Knaak, M. A. \& Peterson, C. L. Histone H3 amino-terminal tail phosphorylation and acetylation: synergistic or independent transcriptional regulatory marks? Cold Spring Harb. Symp. Quant. Biol. 69, 219-226 (2004).
67. Braude-Zolotarjova, T. Y., Kakpakov, V. T. \& Schuppe, N. G. Male diploid embryonic cell line of Drosophila virilis. Vitr. Cell. Dev. Biol. 22, 481-484 (1986).

68. Morales, V., Regnard, C., Izzo, A., Vetter, I. \& Becker, P. B. The MRG domain mediates the functional integration of MSL3 into the dosage compensation complex. Mol. Cell. Biol. 25, 5947-5954 (2005).

69. Fauth, T., Muller-Planitz, F., Konig, C., Straub, T. \& Becker, P. B. The DNA binding CXC domain of MSL2 is required for faithful targeting the dosage compensation complex to the X chromosome. Nucleic Acids Res. 38, 3209-3221 (2010)

70. Punzeler, S. et al. Multivalent binding of PWWP2A to H2A.Z regulates mitosis and neural crest differentiation. EMBO J. 36, 2263-2279 (2017)

71. Bewley, G. C. Drosophila: a practical approach. In Developmental Genetics (ed. Roberts, D. B.) 295 (IRL Press, Oxford-Washington DC, 1987).

72. Zhang, X. et al. Proteome-wide identification of ubiquitin interactions using UbIA-MS. Nat. Protoc. 13, 530-550 (2018).

73. Straub, T., Grimaud, C., Gilfillan, G. D., Mitterweger, A. \& Becker, P. B. The chromosomal high-affinity binding sites for the Drosophila dosage compensation complex. PLoS Genet. 4, e1000302 (2008).

74. Cai, W., Jin, Y., Girton, J., Johansen, J. \& Johansen, K. M. Preparation of Drosophila polytene chromosome squashes for antibody labeling. J. Vis. Exp. https://doi.org/10.3791/1748 (2010).

75. Johansen, K. M. et al. Polytene chromosome squash methods for studying transcription and epigenetic chromatin modification in Drosophila using antibodies. Methods 48, 387-397 (2009).

76. Kamakaka, R. T. \& Kadonaga, J. T. The soluble nuclear fraction, a highly efficient transcription extract from Drosophila embryos. Methods Cell Biol. 44, 225-235 (1994).

77. Cox, J. \& Mann, M. MaxQuant enables high peptide identification rates, individualized p.p.b.-range mass accuracies and proteome-wide protein quantification. Nat. Biotechnol. 26, 1367-1372 (2008).

78. Gatto, L. \& Lilley, K. S. MSnbase-an R/Bioconductor package for isobaric tagged mass spectrometry data visualization, processing and quantitation. Bioinformatics 28, 288-289 (2012).

79. Thomas, P. D. et al. PANTHER: a library of protein families and subfamilies indexed by function. Genome Res. 13, 2129-2141 (2003).

80. Mi, H. et al. PANTHER version 7: improved phylogenetic trees, orthologs and collaboration with the Gene Ontology Consortium. Nucleic Acids Res. 38, D204-D210 (2010).

81. Shannon, P. et al. Cytoscape: a software environment for integrated models of biomolecular interaction networks. Genome Res. 13, 2498-2504 (2003).

82. Szklarczyk, D. et al. STRING v10: protein-protein interaction networks, integrated over the tree of life. Nucleic Acids Res. 43, D447-D452 (2015).

83. Wojcik, F. et al. Functional crosstalk between histone H2B ubiquitylation and H2A modifications and variants. Nat. Commun. 9, 1394 (2018).

84. Fierz, B. et al. Histone H2B ubiquitylation disrupts local and higher-order chromatin compaction. Nat. Chem. Biol. 7, 113-119 (2011).

85. Debelouchina, G. T., Gerecht, K. \& Muir, T. W. Ubiquitin utilizes an acidic surface patch to alter chromatin structure. Nat. Chem. Biol. 13, 105-110 (2017).

86. Luger, K., Rechsteiner, T. J. \& Richmond, T. J. in Chromatin Protocols (ed. Peter B. Becker) 1-16 (Humana Press, 1999).

87. Schauer, T. et al. Chromosome topology guides the Drosophila dosage compensation complex for target gene activation. EMBO Rep. 18, 1854-1868 (2017).

88. Bonte, E. \& Becker, P. B. Preparation of chromatin assembly extracts from preblastoderm Drosophila embryos. Methods Mol. Biol. 119, 187-194 (1999).

89. $\mathrm{Li}, \mathrm{H}$. et al. The sequence alignment/map format and SAMtools. Bioinformatics 25, 2078-2079 (2009).

90. Quinlan, A. R. \& Hall, I. M. BEDTools: a flexible suite of utilities for comparing genomic features. Bioinformatics 26, 841-842 (2010).

91. Langmead, B., Trapnell, C., Pop, M. \& Salzberg, S. L. Ultrafast and memoryefficient alignment of short DNA sequences to the human genome. Genome Biol. 10, R25 (2009).

92. Dobin, A. et al. STAR: ultrafast universal RNA-seq aligner. Bioinformatics 29, 15-21 (2013)

93. Li, B. \& Dewey, C. N. RSEM: accurate transcript quantification from RNASeq data with or without a reference genome. BMC Bioinforma. 12, 323 (2011).

94. Love, M. I., Huber, W. \& Anders, S. Moderated estimation of fold change and dispersion for RNA-seq data with DESeq2. Genome Biol. 15, 550 (2014).

95. Villa, R., Schauer, T., Smialowski, P., Straub, T. \& Becker, P. B. PionX sites mark the X chromosome for dosage compensation. Nature 537, 244-248 (2016).

96. Edgar, R., Domrachev, M. \& Lash, A. E. Gene Expression Omnibus: NCBI gene expression and hybridization array data repository. Nucleic Acids Res. 30, 207-210 (2002). 
97. Deutsch, E. W. et al. The ProteomeXchange consortium in 2017: supporting the cultural change in proteomics public data deposition. Nucleic Acids Res. 45, D1100-D1106 (2017).

\section{Acknowledgements}

We thank A. Lukacs, K. Prayitno, and L. Harpprecht for sharing embryo extracts. We thank A. Scacchetti for establishing the D. virilis spike-in approach. We thank B.V. Andrianov to kindly share D. virilis cell line and J. Kadonaga for NDF reagents. We thank A. Imhof, I. Forné, and M. Wirth at ZFP for mass spectrometry services, S. Krebs and the LAFUGA Genomics Facility for next generation sequencing, T. Straub and the Bioinformatics Unit for providing the high performance computational cluster, E. Kremmer for monoclonal antibody generation, $\mathrm{H}$. Loyd for help with antibody labelings of polytene chromosomes and C. Grimaud for confocal imaging. This work was supported by a grant from the Deutsche Forschungsgemeinschaft to PBB (Be1140/8-1). C.A. acknowledges a DFG fellowship from the Graduate School for Quantitative Biosciences Munich (QBM). Work in the K.M.J. and J.J. laboratory was supported by NIH grant R01 GM62916 and the Roy J. Carver Foundation. National Institutes of Health (NIH) Grants R37 GM086868, R01 GM107047, and P01 CA196539 supported the research in the laboratory of T.W.M. F.W. was funded by a postdoctoral fellowship from the German Research Foundation (WO 2039/1-1).

\section{Author contributions}

C.R. conceived this study and performed experiments. C.A. performed MNase and sonication ChIP-seq experiments and all bioinformatics analysis also with support from T.S. C.W. generated and characterized the cw 2 mutant line with help from J.G., W.C. did the LacO-LacI targeting experiments with support from Y.L., and J.J. and K.M.J. supervised the work and secured funding. G.P.D. performed mononucleosome library experiments and F.W. generated the mononucleosomes and arrays for the kinase assays in TWM's lab. S.K. prepared recombinant proteins for all in vitro assays and RNA-seq libraries under the supervision of C.R. and spike-in ChIP-seq experiments under supervision of C.A. S.M. studied RNA binding by JASPer. P.B.B. secured funding and established collaborations. All authors analyzed data. C.R. and C.A. wrote the paper with contributions from all authors.

\section{Competing interests}

The authors declare no competing interests.

\section{Additional information}

Supplementary information is available for this paper at https://doi.org/10.1038/s41467019-13174-6.

Correspondence and requests for materials should be addressed to P.B.B. or C.R.

Peer review information Nature Communications thanks Elena Casacuberta, Carol Chen and Rakesh Mishra for their contribution to the peer review of this work. Peer reviewer reports are available.

Reprints and permission information is available at http://www.nature.com/reprints

Publisher's note Springer Nature remains neutral with regard to jurisdictional claims in published maps and institutional affiliations.

Open Access This article is licensed under a Creative Commons Attribution 4.0 International License, which permits use, sharing, adaptation, distribution and reproduction in any medium or format, as long as you give appropriate credit to the original author(s) and the source, provide a link to the Creative Commons license, and indicate if changes were made. The images or other third party material in this article are included in the article's Creative Commons license, unless indicated otherwise in a credit line to the material. If material is not included in the article's Creative Commons license and your intended use is not permitted by statutory regulation or exceeds the permitted use, you will need to obtain permission directly from the copyright holder. To view a copy of this license, visit http://creativecommons.org/ licenses/by/4.0/.

(C) The Author(s) 2019 